\title{
Lebecetin, a C-type lectin, inhibits choroidal and retinal neovascularization
}

Fadoua Montassar ${ }^{1,2,3}$, Marie Darche ${ }^{1}$, Amandine Blaizot ${ }^{1}$, Sébastien Augustin ${ }^{1}$, Jean-Baptiste Conart $^{1}$, Aurélie Millet ${ }^{2}$, Mohamed Elayeb ${ }^{2}$, José-Alain Sahel ${ }^{1}$, Annabelle Réaux-Le

Goazigo $^{1}$, Florian Sennlaub ${ }^{1}$, Naziha Marrakchi ${ }^{2}$, Erij Messadi ${ }^{2}$, Xavier Guillonneau ${ }^{*}$.

\section{Author information}

1-Sorbonne Universités, UPMC Univ Paris 06, INSERM, CNRS, Institut de la Vision, 17 rue Moreau, 75012 Paris, France.

2 -Laboratoire des Venins et Biomolécules Thérapeutiques LR11IPT08, Institut Pasteur de Tunis, 13, Place Pasteur, 1002 Tunis, Tunisia

3- Université de Carthage, Faculté des Sciences de Bizerte, Tunisia

\section{* Corresponding author}

Xavier Guillonneau

UMRS 968 Inserm, Institut de la Vision

17, Rue Moreau, 75012 Paris, France

E-mail: xavier.guillonneau@,inserm.fr

Telephone number: +331534626 94, Fax number: +33153462502

Running foot: Lebecetin inhibits ocular neovascularization 


\section{Glossary}

AMD : Age related macular degeneration

CNV : Choroidal neovascularization

DR : Diabetic retinopathy

ERG: Electroretinography

HBMEC : Human brain microvascular endothelial cells

LCT : Lebecetin

NV : Neovascularization

OIR : Oxygen induced retinopathy

OS : Photoreceptor outer segments

OX: Optic chiasm

PDR : Proliferative diabetic retinopathies

PRP : Pan-retinal photocoagulation

RNV : Retinal neovascularization

RPE : Retinal pigment epithelium

SD-OCT : Spectral domain optical coherence tomography

SMP : Subretinal mononuclear phagocytes

VEGF : Vascular endothelial growth factor

VMPO : Ventromedial preoptic nucleus

VO : Vaso-obliteration 


\begin{abstract}
Angiogenesis is a cause of visual impairment and blindness in the wet form of Age related Macular Degeneration (AMD) and in ischemic retinopathies. Current therapies include the use of anti-VEGF agents to reduce choroidal neovascularization and edema. These treatments are effective in most cases, but spontaneous or acquired resistance to anti-VEGF and possible advert effects of long-term VEGF inhibition in the retina and choroid highlight a need for additional alternative therapies. Integrins $\alpha v \beta 3$ and $\alpha v \beta 5$ that regulate endothelial cell proliferation and stabilization have been implicated in ocular angiogenesis. Lebecetin (LCT) is a $30 \mathrm{kDa}$ heterodimeric C-type lectin isolated from Macrovipera lebetina venom that interacts with $\alpha 5 \beta 1$ and $\alpha \mathrm{V}$-containing integrins. We previously showed that LCT inhibits human brain microvascular endothelial cells (HBMEC) cell adhesion, migration, proliferation and tubulogenesis. To evaluate the inhibitory effect of LCT on ocular angiogenesis, we cultured aortic and choroidal explants in the presence of LCT and analyzed the effect of LCT on choroidal neovascularization in the mouse CNV model and on retinal neovascularization in the oxygen induced retinopathy (OIR) model. Our data demonstrated that a single injection of LCT efficiently reduced choroidal and retinal neovascularization in these models.
\end{abstract}

Key words: Integrins, C-type lectin, Snake venom, Retinopathy, Angiogenesis. 


\section{Introduction}

Age related Macular degeneration (AMD) is the leading cause of blindness in people over 55 years of age, and ischemic retinopathies such as diabetic retinopathy (DR), retinal vein occlusion and retinopathy of prematurity, is the leading cause of blindness in people under 55 years of age (1-3). Proliferative forms of these pathologies (wet AMD and proliferative diabetic retinopathy) result in rapid and non-reversible vision loss. In AMD, new vessels mainly originate from the vascular choroidal bed and grow in the subretinal space or underneath the retinal pigment epithelium (RPE) while in the proliferative form of DR (PDR), neural ischemia trigger neovascularization from the retinal vessels. Vascular endothelial growth factor (VEGF) is a major mediator of retinal and choroidal angiogenesis (4). Intraocular injections of antibodies directed against VEGF or of soluble form of VEGFR1 efficiently inhibit choroidal neovascularization in wet AMD. However $10 \%$ of the treatmentnaïve patients do not respond to anti-VEGF $(5,6)$ and 2 to $10 \%$ of the anti-VEGF responders become resistant with time $(7,8)$. Anti-VEGF therapies are also the first line treatment of diabetic macular edema. In contrast, PDR that are characterized by retinal neovascularization (RNV) are mostly treated by a preventive pan-retinal photocoagulation (PRP) (9). AntiVEGFs are now approved in the US for the treatment of PDR as an alternative to PRP. Ongoing studies will determine the rate of spontaneous and acquired resistance in this new indication. All together, these clinical data support the need for additional therapies that do not primarily target the VEGF pathway.

Integrins are receptors of specific extracellular matrix proteins that have been implicated in angiogenesis both as positive or negative regulators of endothelial cell proliferation and stabilization (10). Integrins $\alpha v \beta 3$ and $\alpha v \beta 5$ are thought to be critical regulators of angiogenesis. $\alpha v \beta 3$ is not expressed on quiescent microvessels but is dramatically increased in response to angiogenic growth factors (11) and $\alpha v \beta 5$ has been shown to be necessary to regulate the angiogenesis mediated by VEGF (12). Both integrins are upregulated during pathological ocular neovascularization $(13,14)$. Cyclic peptides mimicking the RGD binding, motif of integrin $\alpha v \beta 3$, antibodies directed against integrin subunit and molecules isolated from venoms have been shown to disrupt integrin/basal membrane recognition and have been used in animal models of cancer, fibrosis and inflammation (15). Their systemic or local (intravitreal) administration has been shown to inhibit choroidal neovascularization (CNV) in laser induced CNV model and retinal neovascularization in the mouse oxygen induced retinopathy (OIR) model (16-21). 
Lebecetin (LCT) is a $30 \mathrm{kDa}$ heterodimeric C-type lectin isolated from Macrovipera lebetina (M. lebetina) venom (22). LCT interacts with $\alpha 5 \beta 1$ and $\alpha$ v-containing integrins (23).

In vitro, LCT inhibits human brain microvascular endothelial cells (HBMEC) cell adhesion, migration, proliferation and tubulogenesis (24). In vivo, LCT reduces angiogenesis in a chick CAM assay but interestingly, unlike lebectin, another C-type lectin, LCT was unable to antagonize FGF2-induced angiogenesis in a matrigel plug assay (24).

We here examined the effect of LCT on angiogenesis in aortic and choroidal cultured explants and compared its activity to Aflibercept, a chimeric VEGF Receptor that inhibit VEGF, in vivo in the $\mathrm{CNV}$ and OIR mice models. Our data demonstrate that a single injection of LCT efficiently reduces the extent of choroidal or retinal neovascularization in these models. 


\section{Materials and Methods}

\section{Animals}

Three and eleven weeks-old C57BL/6JRj male mice and 4-days-old Lewis rat pups were purchased from Janvier Labs (Le Genest- Saint-Isle, France). Eleven weeks-old CX3CR1 ${ }^{+} / \mathrm{GFP}$ male mice were obtained from the Jackson Laboratory (Bar Harbor, USA). Animals were housed in the animal facility under specific pathogen-free condition, in a 12/12 h light/dark cycle with water and normal diet food available ad libitum.

All procedures were performed in accordance with the guidelines from Directive 2010/63/EU of the European Parliament on the protection of animals used for scientific purposes and approved by the Institutional Animal Care and Use Committee, Comité d'éthique pour l' expérimentation animale Charles Darwin ( $\mathrm{N}^{\circ}$ 02371.02).

\section{Vascular sprouting from aortic ring ex vivo}

After decapitation of Lewis rat pups, thoracic aortas were cut into 1-mm-thick rings and covered with $15 \mu \mathrm{l}$ of growth factor-reduced phenol red free matrigel (Corning, Boulogne Billancourt, France) in 48 well tissue culture plates. Aortic rings were cultured for 3 days in Dulbecco's Modified Eagle's Medium (DMEM) (Thermo Fisher Scientific, Villebon-surYvette, France) supplemented with 10\% fetal bovine serum, 1\% penicillin/streptomycin, and $0.2 \%$ fungizone (25). Explants were exposed to LCT at different doses (30 nM, $300 \mathrm{nM}, 1.5$ $\mu \mathrm{M}$ ) from day 3 (D3) to D6 of culture. Control explants were cultured in DMEM without addition of LCT. Photographs of individual explants were taken from D3 to D6. The surface of each individual aortic ring and pre-incubation sprouts at D3 was subtracted from the surface at D6 to calculate the vascular sprouting that occurred in the presence or absence of LCT. The areas of sprouting were quantified with Fiji software (28). Data are expressed as the percentage of growth between D6 and D3.

\section{Vascular sprouting from choroid ex vivo}

Eyes were enucleated from C57BL/6JRj mice and kept in ice-cold endothelium growth medium (EGM-2) (Lonza, Levallois-Perret, France) before dissection. Choroid was separated from the other eye tissues and cut into approximately $1 \mathrm{~mm} \times 1 \mathrm{~mm}$. Choroid fragments were isolated and placed in growth factor-reduced phenol red free matrigel (Corning, Boulogne Billancourt, France) seeded in 48 well plates. 
Choroidal explants were then cultured for 3 days in EGM-2 medium supplemented with 5\% fetal bovine serum, $1 \%$ penicillin/streptomycin, and $0.2 \%$ fungizone in a $37^{\circ} \mathrm{C}$ cell culture incubator (26). On D3, Choroid fragments were treated with LCT (1.5 nM, $5 \mu \mathrm{M}, 15 \mu \mathrm{M})$ from D3 until D6 of culture. Photos of individual explants were taken and the areas of sprouting were quantified with Fiji software (28). The surface of each individual choroidal explant and pre-incubation sprouts at D3 was subtracted from the surface at D6 to calculate the vascular sprouting that occurred in the presence or absence of LCT.

\section{SD-OCT}

Pupils were dilated with tropicamide (Mydriaticum) Théa, Clermont-Ferrand, France) and phenylephrin (Neosynephrine) (Europhta, Monaco). Mice were then anesthetized by inhalation of isoflurane (2\%) (Axience, Pantin, France) and placed in front of the spectral domain optical coherence tomography (SD-OCT) imaging device (Bioptigen $840 \mathrm{~nm}$ HHP; Bioptigen, North Carolina, USA). Images were acquired from optic disc at approximately 0.1 or $1.4 \mathrm{~mm}$ of the superior retina. SD-OCT was calibrated $(1 \mathrm{pixel}=1.6 \mu \mathrm{m})$ as previously described (27). Retinal layer, inner nuclear layer (INL), outer nuclear layer (ONL) and photoreceptor outer segments (OS) thicknesses were measured at $500 \mu \mathrm{m}$ from the centre of the optic nerve at day 7 by FIJI software (28).

\section{Electroretinography (ERG)}

ERG was performed 7 days after injection of PBS and LCT (500 $\mu \mathrm{M})$. C57BL/6JRj mice were kept overnight for dark adaptation and then anesthetized with an intraperitoneal injection of ketamine (100 mg/kg, Virbac, Carros, France) and xylazine (10 mg/kg, Bayer HealthCare, Berlin,Germany). Pupils were dilated with phenylephrin (Neosynephrine) (Europhta, Monaco) and tropicamide (Mydriaticum) (Théa, Clermont-Ferrand, France). The cornea was anesthetized with oxybuprocaine chlorhydrate (Théa, Clermont-Ferrand, France). Body temperature was maintained at $37^{\circ} \mathrm{C}$ using a heating pad. Upper and lower lids were retracted to keep eyes open and bulging. A gold-loop electrode was placed in contact with the surface of each cornea and maintained with lubrithal (Zubial, Auros, France) to record ERG (Espion, Diagnosys LLC, Lowell, MA, USA). Reference and ground electrodes were respectively placed in the forehead and in the back of animal. The light stimulus was provided by Ganzfeld stimulator (Espion, Diagnosys LLC, Lowell, MA, USA). Responses were amplified and filtered (1 Hz-low and $300 \mathrm{~Hz}$-high cut off filters) with a 1 channel DC/AC-amplifier. Five levels of stimulus intensity $\left(0.003 \mathrm{~cd} . \mathrm{s} / \mathrm{m}^{2} ; 0.03 \mathrm{~cd} . \mathrm{s} / \mathrm{m}^{2} ; 0.3 \mathrm{~cd} . \mathrm{s} / \mathrm{m}^{2} ; 3 \mathrm{~cd} . \mathrm{s} / \mathrm{m}^{2} ; 10 \mathrm{~cd} . \mathrm{s} / \mathrm{m}^{2}\right)$ 
were used for scotopic ERG recording. Each scotopic ERG response represents the average of five responses from a set of five flashes of stimulation.

To evaluate cone responses, mice were exposed 5 minutes to the light at $20 \mathrm{~cd} / \mathrm{m}^{2}$ to saturate rod photoreceptors. A $10 \mathrm{~cd} . \mathrm{s} / \mathrm{m}^{2}$ level of stimulus intensity was used for the light adapted ERGs. The light adapted ERGs were recorded on the same rod-suppressive white background as for the light adaptation. Each cone photopic ERG response represents the average of ten responses to a set of ten consecutive flashes. The flicker ERG was also used to isolate cone responses at flash frequencies of 10 and $20 \mathrm{~Hz}$ at $1 \mathrm{~cd} . \mathrm{s} / \mathrm{m}^{2}$ intensity.

\section{Laser-induced choroidal neovascularization (CNV) model}

$\mathrm{C} 57 \mathrm{BL} / 6 \mathrm{JRj}$ mice were anesthetized with an intraperitoneal injection of ketamine (100 $\mathrm{mg} / \mathrm{kg}$, Virbac, Carros, France) and xylazine (10 mg/kg, Bayer HealthCare, Berlin,Germany). Pupils were dilated and 4 laser coagulations (400 mW, $50 \mathrm{~ms}, 100 \mu \mathrm{m}$ spot size) were performed with a Laser Yag 532 Eyelite (Alcon, Rueil-Malmaison, France) mounted on a slit lamp (BQ 900, Hagg-Streitt, Chambery, France). Laser photocoagulation and rupture of Bruch's membrane were confirmed by immediate observation of a bubble $(25,29)$. Mice were injected with $1 \mu \mathrm{l}$ of PBS, LCT $(500 \mu \mathrm{M})$ or Aflibercept $(25 \mu \mathrm{M})$ immediately or 3 days after laser.

7 days after lesion, retinas of mice were examined with SD-OCT. OCT sequences were acquired and analyzed with Fiji (28). Lesion volume was calculated with the formula $\left(4 / 3 \pi^{*} a^{*} b^{2}\right) / 2$, where $a$ is the polar radius that corresponds to the measure along the vertical axis and $\boldsymbol{b}$ is the equator radius that corresponds to the horizontal axis (29).

On D7, mice were euthanized by CO2 inhalation and CNV areas were quantified on immunostained choroidal flatmounts with MetaMorph software (Molecular Devices, SaintGregoire, France).

\section{Oxygen-induced retinopathy (OIR) model}

C57BL/6JRj pups mice with nursing mothers were exposed to $75 \%$ oxygen at postnatal day (P) 7 for 5 consecutive days as previously reported (30). On P12, mice were returned to roomair and injected intravitreally with PBS, LCT $(500 \mu \mathrm{M})$ or Aflibercept $(25 \mu \mathrm{M})$. At P17 mice were sacrificed by $\mathrm{CO}_{2}$ inhalation and retinas were dissected. Vaso-obliteration (VO) and neovascularization $(\mathrm{NV})$ areas were calculated on flatmounted immunostained retinas with MetaMorph software (Molecular Devices, Saint-Gregoire, France). 


\section{RT-qPCR}

Integrin subunits $\alpha v, \alpha 5, \beta 3$ and $\beta 5$ gene expression was quantified by reverse transcription quantitative polymerase chain reaction (RT-qPCR) in CNV model at days 0, 1, 3 and 7 after laser injury. Choroids were dissected in RNase-free conditions. Total RNA was isolated with Nucleospin RNAII (Macherey Nagel, Hoerdt, France). Single-stranded cDNA was synthesized from total RNA (pretreated with DNaseI amplification grade, Thermo Fisher Scientific, Villebon-sur-Yvette, France) using oligo-dT as a primer and superscript II reverse transcriptase (Thermo Fisher Scientific, Villebon-sur-Yvette, France). Real-time polymerase chain reaction was performed using cDNA and SYBR Green Gene Expression Master Mix (Thermo Fisher Scientific, Villebon-sur-Yvette, France) and the following primers (0.5 pmol/ $\mu$ l) (Life Technologies, Saint-Aubin, France): GAPDH sense: 5'-ACG GCC GCA TCT TCT TGT GCA-3'; GAPDH antisense: 5'-CAG GCG CCC AAT ACG GCC AA-3'; ITGAV sense: 5'-CAC CCT CAG AGA GGG AGA TG-3'; ITGAV antisense: 5'-ACG TAC AGG ATT GCG CTC TT-3'; ITGA5 sense: 5'-AGT ACG CAC CTT GCC GCT CA-3'; ITGA5 antisense: 5'-ACA CGG CCA GTC TTG GTG AAC-3'; ITGB3 sense: 5'-AAC CGG GGA ACG CTC CAT GA-3'; ITGB3 antisense: 5'-CGG CGT TTT TGC CAG TAT CCG-3'; ITGB5 sense: 5'-AGC CTT TGG GGA GAC GTG TGA-3'; ITGB5 antisense: 5'-TGG TGG TGG CAG GTC TGG TT-3'.

PCR reactions were performed in 45 cycles of $15 \mathrm{~s}$ at $95^{\circ} \mathrm{C}, 45 \mathrm{~s}$ at $60^{\circ} \mathrm{C}$. Data were normalized to GAPDH and expressed relative to control group values.

\section{Reagents and drugs}

LCT was obtained as previously described (22). Briefly, venom of M.lebetina was gelfiltrated using Sephadex G-75 Column. Firstly, LCT was purified by FPLC on a Mono S (HR5/5) column and eluted with linear 0-1 M NaCl gradient. LCT was lyophilized and next dissolved in PBS. LCT preparation quality was tested on C8 reversed phase HPLC column with linear gradient of acetonitrile (22). Aflibercept (Eylea; Bayer, Lyon, France) was kindly provided by Dr Chiara Eandi (University of Torino) and Dr Audrey Giocanti-Aurégan (Hopital Avicenne Paris). For in vivo studies $1 \mu \mathrm{l}$ of the following solution were injected in the vitreous: $500 \mu \mathrm{M} \operatorname{LCT}(15 \mu \mathrm{g} / \mu \mathrm{l})$ or $25 \mu \mathrm{M}$ Aflibercept $(2,5 \mu \mathrm{g} / \mu \mathrm{l})$. In some experiments, $2 \mu 1$ of PBS or of labeled-LCT, -Aflibercept or -BSA were injected in the right eye. 


\section{Labeling proteins with Alexa Fluor ${ }^{\circledR} 647$}

Alexa Fluor ${ }^{\circledR} 647$ microscale protein labeling Kit (Thermo Fisher Scientific, Villebon-surYvette, France) was used to label LCT $(500 \mu \mathrm{M})$, Aflibercept $(25 \mu \mathrm{M})$ and Bovine Serum Albumin (BSA, $15.4 \mu \mathrm{M}$ ). Proteins were dissolved in $1 \mathrm{M}$ sodium bicarbonate and mixed with Alexa Fluor 647 succinimidyl ester that react with primary amines of proteins and incubated for $1 \mathrm{~h}$ at $4{ }^{\circ} \mathrm{C}$. The conjugated protein was separated from unreacted dye using the supplied spin column at room temperature. The final concentration was estimated according to manufacturer's recommendations to $1 / 4$ of the initial concentration.

\section{Histological analysis}

7 days after CNV and injection of PBS or 647-LCT, a $300 \mu \mathrm{L}$ mixture of ketamine (100 $\mathrm{mg} / \mathrm{kg}$, Virbac, Carros, France) and xylazine (10 mg/kg, Bayer HealthCare, Berlin,Germany) was injected intraperitoneally to deeply anesthetized animals. Mice were perfused via the ascending aorta with $5 \mathrm{~mL}$ of $0.9 \% \mathrm{NaCl}$ solution followed by $30 \mathrm{~mL}$ of $4 \%$ paraformaldehyde solution. After fixation, brain was carefully dissected out and post-fixed 48 $\mathrm{h}$ in the same fixative. Free-floating sections $(40 \mu \mathrm{m})$ were performed using a vibratome (Leica Microsystems, Wetzlar, Germany).

\section{Immunochemistry}

Mice were euthanized by CO2 inhalation. Eyes were enucleated and fixed in 4\% paraformaldehyde for $30 \mathrm{~min}$ at room temperature. After several washes in PBS, the cornea and lens were removed and the retina was carefully separated from RPE/choroid/sclera. Retinal flatmounts were stained with goat polyclonal anti-collagen IV antibody (AbD Serotec, Cergy Pontoise, France) and FITC-coupled Bandeirae simplicifolia (BS)-1 lectin (SigmaAldrich, Saint Quentin Fallavier, France). Astrocytes and activated Muller cells were labeled using anti-Glial Fibrillary Acidic Protein (GFAP) antibody (Sigma-Aldrich, Saint Quentin Fallavier, France) and microglial cells were stained using rabbit polyclonal anti-Iba1 (Wako, Neuss, Germany). The RPE was stained using TRITC-coupled phalloidin (Sigma-Aldrich, Saint Quentin Fallavier, France) on choroidal flatmounts. Nuclei were stained with DAPI (Sigma-Aldrich, Saint Quentin Fallavier, France). In CNV model, neovessels were immunostained with CD102 (Rat anti-mouse, BD Biosciences Pharmingen, Le Pont de Claix, France), microglial cells were labeled using anti-Ibaland endothelial cells nuclei were stained with DAPI on choroidal flatmounts. Brain sections were placed in a blocking solution containing 3\% Normal Goat Serum and $0.1 \%$ triton X-100 for $1 \mathrm{~h}$, then incubated with rabbit 
anti-ATF3 (Santa Cruz Biotechnology, Heidelberg, Germany) and TRITC-coupled Bandeirae simplicifolia (BS)-1 lectin at $4^{\circ}$ for $48 \mathrm{~h}$ and stained with DAPI. In OIR model, retinal capillaries were labeled with FITC-BS-1 lectin. The corresponding Alexa-conjugated secondary antibodies (Thermo Fisher Scientific, Villebon-sur-Yvette, France) were used to reveal the primary antibodies.

Retina, choroids and brain sections were viewed with a fluorescence microscope (DM5500B) (Leica, Saint Jorioz, France) or with a confocal microscope (FV1000) (Olympus, Rungis, France). The microscope was calibrated for control mice (PBS) before acquisitions in LCTinjected mice.

\section{Statistical analysis}

GraphPad Prism (GraphPad Software, San Diego, USA) was used for data analysis and graphic representation. All values are reported as mean \pm SEM. Data were analyzed by MannWhitney U test, one-way ANOVA followed by Bonferroni or Dunnett's post-tests. $\mathrm{P}<0.05$ was considered as statistically significant. 


\section{Results}

\section{LCT inhibits vascular sprouting from aortic and choroidal explants}

LCT inhibits endothelial cell proliferation and tubulogenesis in vitro (24). To test if LCT inhibits angiogenesis ex vivo, we cultured mouse aortic rings in matrigel (25). Aortic rings were cultured for 3 days to allow for vessel sprouting and then treated with increasing doses of LCT. Three days after the addition of LCT, vessel sprouting area was quantified and expressed as the increase (in percentage) of sprouting area between D3 and D6 (Fig. 1A and B). In control conditions, vascular sprouting increased by $259 \%$ between D3 and D6. LCT added at a final concentration of $30 \mathrm{nM}$ did not affect vascular sprouting while $300 \mathrm{nM}$ of LCT reduced vascular sprouting between D3 and D6 to 85\%. LCT at a dose of $1.5 \mu \mathrm{M}$ totally inhibited sprouting and resulted in the regression of pre-existing D3 vascular sprouts (Fig. 1B). Increasing doses of LCT did not notably affect fibroblasts that grow out of the explant and proliferate on the plastic dish cell surface. LCT activity was next tested in the mouse choroidal explant model that closely reproduces the formation of vessels from the choriocapillary bed (26). Choroids were cultured as explants as previously described (26). As for aortic rings, explants were treated at D3 with LCT and analyzed at D6 (Fig. 1C and D). We first used the dose that resulted in vascular regression in aortic rings. At $1.5 \mu \mathrm{M} \mathrm{LCT}$ inhibited vessel sprouting by $78 \%$ when compared to control conditions but still allowed for a $286 \%$ increase of vessels compared to D3. At $5 \mu \mathrm{M}$, LCT efficiently inhibited vessel growth but failed at regressing pre-existing D3 vascular sprouts. Finally, at $15 \mu \mathrm{M}$, LCT induced D3 sprouts regression (Fig. 1D). LCT was thus effective at reducing vascular sprouting in two independent ex vivo models of neovascularization.

\section{LCT intravitreal injection does not alter retinal integrity}

Ex vivo experiments demonstrated variability in the dose required to inhibit neovascularization. We thus ran a pilot study to determine the concentration required to reduce neovascularization in the model of laser induced choroidal neovascularization (CNV). One $\mu 1$ of LCT was used for intravitreal injection, as the vitreous volume is $5.3 \mu 1$ (31), the initial concentration of LCT could be estimated to $1 / 5$ of its initial concentration. Depending on its pharmacokinetics LCT may then reaches all ocular compartments $(10 \mu 1)$ and its concentration may decrease to $1 / 10$ of the initial concentration. Animals were thus intravitrealy injected with $1 \mu \mathrm{l}$ of 150 or $500 \mu \mathrm{M}$ of LCT to reach the estimated final concentration of $15 \mu \mathrm{M}$ (the dose that regress choroidal explant sprouts) or $50 \mu \mathrm{M}$ (the 
highest concentration we can purify from venom). We determined that an intravitreal injection of $1 \mu \mathrm{LCT}$ at $150 \mu \mathrm{M}$ was not sufficient to reduce CNV while a $1 \mu \mathrm{l}$ injection of $500 \mu \mathrm{M}$ LCT decreases the neovascular area. To test if a single injection of $500 \mu \mathrm{M}$ LCT alters retinal architecture we injected LCT in control adult mice and examined their retina after 7 days. Retinal architecture was analyzed by SD-OCT. OCT did not reveal significant changes in the overall structure of the retina (Fig. 2A). The thickness of the entire retina, of the inner and outer nuclear layers and outer segments was not statistically different between controls and LCT injected-eyes (Fig. 2B). ERG responses were recorded from control animals and treated animals 7 days after injection. Intravitreal injections of LCT did not alter scotopic ERG recorded at intensity ranging from 0.003 to $10 \mathrm{cds} / \mathrm{m}^{2}$ (Fig. 2C; D) when compared to control or PBS-injected animals. Similarly photopic responses (Fig. 2E) and flicker responses (Fig. 2F; G) were not altered after LCT injections when compared to control animals. Vasculature integrity was next evaluated by immunochemistry 7 days after injection. Retinal flatmounts were stained with FITC-BS-1 lectin and collagen-IV that label respectively endothelial cells and vascular basal membranes. Ghost vessels (detected as collagen IVpositive, lectin-negative vessels) and neovascular tufts were not detected in eyes injected with LCT at $500 \mu \mathrm{M}$ (Fig. 3A). Loss of vascular integrity results in micro- and macro-glial cell activation. Retinal flatmounts were thus immunostained with anti-GFAP (specific for astrocytes and activated Muller cells) or anti-Iba1 (specific for microglial cells) antibodies. LCT did not modify astrocyte morphology and vessel coverage and no sign of Muller cell activation was found in the inner layer of the retina one week after LCT or PBS intravitreal injection (Fig. 3B). We next examined microglial cells morphology after LCT injection in the vitreous of CX3CR1 $1^{+/ G F P}$ mice. LCT did not modify the morphology of CX3CR1-positive cell located in the superficial or deep plexus (Fig. 3C). RPE cell morphology was assayed using TRITC-coupled phalloidin, 7 days after LCT injection. No sign of RPE cells death or alteration of RPE morphology was detected (Fig. 3D). All together our results indicated that a LCT does not alter retinal architecture, retinal function, or vessel integrity 7 days after injection.

\section{LCT inhibits laser-induced choroidal neovascularization}

We showed that LCT inhibits HBMEC proliferation and tubulogenesis in vitro (24) and vascular sprouting in ex vivo models of neovascularization (Fig. 1) without affecting vascular integrity (Fig. 3). To test if LCT inhibits in vivo angiogenesis, LCT activity was assayed in the CNV mouse model. Integrin subunits $\alpha \mathrm{v}$ and $\alpha 5$ has been shown recently to be increased 
from D3 to D7 in the rat CNV model (32). We quantified the expression of integrin subunits $\alpha v$ and $\alpha 5$ and $\beta 3$ and $\beta 5$ in mouse choroids after laser-induced choroidal lesions at different time-points. Choroidal lesions were induced on D0 with an ophthalmic laser and choroids were collected at D1, D3 and D7. The expression of these subunits was analyzed by RTqPCR and compared to non-lesioned choroids (D0). The expression of all subunits was found to be increased within $24 \mathrm{~h}$ after lesion and peaked at D3. At D7, $\alpha \mathrm{v}$ returned to basal level while $\alpha 5, \beta 3$ and $\beta 5$ remained elevated (Fig. 4A). To determine the specificity of LCT binding after intravitreal injections, we next injected labeled molecules in the vitreous of laserlesioned eyes. Bovine serum albumin (BSA), LCT and Aflibercept were covalently conjugated to an Alexa Fluor 647 dye using a microscale protein labeling kit, and then purified and injected three days (D4) before sacrifice (D7) in the right eye. Left eye received a PBS injection. Alexa Fluor 647-conjugated BSA (647-BSA) did not labeled CD102-postive CNV lesions. In contrast both 647-LCT and 647-Aflibercept labeling was found in CD102positive CNV lesions on choroidal flatmount (Fig. 4D; E). Retinal flatmounts were then labeled with Collagen-IV to detect vascular basal membranes and deposits. An intense 647LCT labeling was found in the outer part of the retina facing the CNV lesion and a faint labeling was observed on large arteries in the LCT-injected eye. No labeling was found in the contralateral PBS-injected eye (suppl. Fig. 1). We next analyzed 647-LCT labeling in the optic nerve, the trigeminal nerve and in different areas of the brain of 647-LCT injected animals and compared it to PBS-only injected animals. LCT was not detected in the the optic and trigeminal nerve (data not shown) nor in hippocampus, piriform cortex, cingulate cortex, hypothalamus, ventromedial preoptic nucleus (VMPO) and the caudate putamen (striatum). Furthermore we did not detect ATF3 labeling indicative of neuronal injury (suppl. Fig. 2) (33, $34)$.

To determine the in vivo anti-angiogenic properties of LCT, we quantified lesion volumes 7 days after laser impact as previously described (29) after a single injection of LCT on D0 and compared it to PBS treated animals. LCT injection decreased the lesion volume by $31.9 \%$ when compared to controls (Fig. 5A). We next quantified the area covered by neovessels on choroidal flatmounts stained with CD102 at D7. CNV areas were significantly reduced by 26.7\% 7 days after LCT injection (Fig. 5B; C). Anti-VEGF are routinely used to treat choroidal neovascularization in AMD patients (35). We next compared LCT to anti-VEGF. Bevacizumab and Ranibizumab commercial antibodies only recognize human VEGF, we thus used Aflibercept that binds both human and mouse VEGF (36). A single injection of 
Aflibercept reduced choroidal neovascularization by $31.5 \%$ (Fig. 5B; C). LCT and Aflibercept-treated lesions were not statistically different in size (Fig. 5A). Subretinal mononuclear phagocytes (sMP) participate in choroidal neovascularization (37). To evaluate the effect of LCT on sMP accumulation around lesion, choroidal flatmounts were stained with the anti-Ibal antibody that labels sMP. No difference in the number of sMP around lesion was found between LCT and PBS treated eyes. Similarly no difference in the number of sMP was found between LCT- and Aflibercept-treated lesions (Fig. 5B; D).

Integrins $\alpha v \beta 3$ and $\alpha v \beta 5$ are critical regulators of angiogenesis, we thus determined if LCT injection regulates their expression. As $\alpha v \beta 3$ and $\alpha v \beta 5$ are expressed by proliferating endothelium, we investigate the expression of $\alpha \mathrm{v}, \alpha 5, \beta 3$ and $\beta 5$ shortly after LCT injection (24h) to discriminate a possible transcriptional regulation from the long term anti-angionic effect of LCT. $\alpha \mathrm{v}, \alpha 5, \beta 3$ and $\beta 5$ expression is maximum at D3, we thus analyze their expression on D3 after a single injection of LCT or PBS on D2. qPCR analysis did not reveal significant differences in their level of expression between LCT and PBS in RPE/choroids extracts (Fig. 5E).

We showed that high doses of LCT regresses existing vessels (Fig.1), we thus next injected LCT in the vitreous three days after laser impacts and quantified the neovascularization size. When injected after the initial vessel growth, LCT injection allowed vascular regression by 19\% (Fig. 6A; B). All together our results showed that a single injection of LCT allowed prevention and regression of choroidal neovascularization without impairing sMP recruitment.

\section{LCT inhibits retinal neovascularization in the oxygen induced retinopathy model}

We next tested the effect of LCT on retinal neovascularization, a hallmark of severe ischemic retinopathies, in the OIR model. This model has been widely used to understand retinal vascular loss, vascular regrowth, neovascularization and neovascular regression (30). Retinal neovascularization is induced by subjecting newborn rodents to hyperoxia between P7 and P12 to inhibit physiological vascular development. When animals are returned to room air, the relative retinal hypoxia leads to severe retinal neovascularization between P12 and P17 (38). To determine the binding specificity of LCT, we first injected Alexa Fluor 647-conjugated LCT (647-LCT) in the vitreous of OIR-subjected animals three days before their sacrifice on P17. 647-LCT intensively labeled BS-1 lectin positive neovessel tufts while retinal vasculature was not labeled (Fig. 7A). To evaluate the anti-angiogenic properties of LCT in 
the OIR model, P7 mice were subjected to OIR and injected with $1 \mu 1$ of PBS or $500 \mu \mathrm{M}$ LCT on day 12 and were returned to room-air. Mice were sacrificed at P17 and vaso-obliteration (VO) and neovascularization area (NV) were determined on retinal flatmount stained with BS-1 lectin as previously described (39). LCT did not change the ratio of VO when compared to PBS injection (Fig 7B; D). In contrast, a single injection of LCT at P12 reduced the area covered by retinal neovascularization at P17 by $48.1 \%$ (Fig 7B; E). To compare LCT treatment to the anti-VEGF therapy, $25 \mu \mathrm{M}$ of Aflibercept was injected at P12, a dose that has been shown to reduce NV without affecting retinal development (39) and NV and VO were compared to LCT animals. No differences were found in VO and NV between LCT and Aflibercept treatments (Fig. 7B-E). 


\section{Discussion}

AMD and ischemic retinopathies are characterized by devastating angiogenesis. Lowering the biodisponibility of VEGF in the vitreous reduces choroidal and retinal neovascularization and edema formation. In the past decade, anti-VEGF have appeared as an effective tool to lower VEGF in the vitreous of AMD and DR patients. However, while effective in a majority of patients, cases of spontaneous resistance or acquired resistance leave some patients without treatment $(7,8)$. Integrins are receptors for specific extracellular matrix proteins and have been implicated in angiogenesis both as positive or negative regulators of endothelial cell proliferation and stabilization (10) and have thus appeared as an attractive target to reduce neovascularization. Pharmacologic inhibition of integrins was attempted in animal models of cancer, fibrosis and inflammation (15). Three classes of integrin inhibitors are in trials: monoclonal antibodies that target the extracellular domain of the integrin heterodimer, synthetic RGD motif-containing peptides, and peptidomimetics that mimic the RGD sequence (41). RGD-mimetic integrin inhibitors have agonistic properties at low concentrations (42, 43) that may limit their use as a therapeutic tool. We have recently isolated Lebecetin (LCT) a $30 \mathrm{kDa}$ heterodimeric C-type lectin from M. lebetina venom (22). LCT interacts with $\alpha 5 \beta 1$ and $\alpha \mathrm{v}$-containing integrins in an RGD-independent manner with $\mathrm{IC}_{50}$ ranging from 400 to $1300 \mathrm{nM}$ (23). In vitro, LCT inhibits HBMEC cell adhesion, migration, proliferation and tubulogenesis (24).

To evaluate the inhibitory effect of LCT on angiogenesis we cultured choroidal explants, a representative model of microvascular ocular angiogenesis (26). We showed an inhibition of $78 \%$ of choroidal vessel sprouting with a concentration of $1.5 \mu \mathrm{M}$ of LCT suggesting that doses within the range of the $\mathrm{IC}_{50}$ are sufficient ex vivo to inhibit choroidal neovascularization. At $15 \mu \mathrm{M}$ (approx. 10x $\mathrm{IC}_{50}$ ) the inhibition of integrins resulted in the regression of preexisting choroidal vascular sprouts (Fig.1). As inhibition of $\alpha 5 \beta 1$ has been shown to induce endothelial cell apoptosis (44), it is likely that high doses of LCT promote $\alpha 5 \beta 1$-dependent apoptosis in endothelial cells, and that it contributes to the anti-angiogenic activity of LCT. In vivo injection of $1 \mu \mathrm{l}$ of $150 \mu \mathrm{M}$ LCT, which is predicted to dilute by a factor of 10 within the eye volume to a concentration of $15 \mu \mathrm{M}$ did not have a significant effect on CNV. CNV prevention and regression was only obtained with a single injection of 1 $\mu 1$ of $500 \mu \mathrm{M}$, which represents an estimated intra-ocular concentration of 50x IC 50 .

The differences between LCT and Aflibercept concentrations (500 vs $25 \mu \mathrm{M})$ needed to reach neovascularization inhibition might reflect that (1) LCT has a much higher $\mathrm{IC}_{50}$ for its targets than Aflibercept for mouse VEGF $(400-1300 \mathrm{nM}$ vs $10 \mathrm{pM})(23,36)$ and (2) that LCT binds 
to multiple highly expressed membrane anchored and extracellular matrix targets while Aflibercept only binds to low expressed extracellular cytokines.

We have shown that LCT binds and inhibits $\alpha 5 \beta 1$ and $\alpha v$-integrins ( $\alpha v \beta 3, \alpha v \beta 5$ and $\alpha v \beta 6)$. These complexes have been shown to be expressed specifically in newly formed vessels (45, 46), suggesting that they could be targeted be safely in vivo to inhibit neovascularization. However, to unambiguously determine LCT binding sites in healthy and diseased retina, we cross-linked LCT to an Alexa Fluor 647 fluorescent dye and injected it into control, CNV or OIR-eyes. Our data demonstrate that 647-LCT strongly labels CNV lesions and OIRneovascular tufts. We did not find LCT fluorescence in the optic or trigeminal nerves nor in the contralateral eye indicating that LCT did not exit the injected eye. In line with that, 647LCT was not detected in the structure observed in the brain (hippocampus, piriform cortex, cingulate cortex, hypothalamus, VMPO and caudate putamen (striatum)). ATF3 labeling did not reveal any neuronal injury in these brain structures analyzed (suppl. Fig. 2).

Binding to non-neovascular structures (as seen with large arteries (suppl. Fig.1)) might result in unexpected off-target effects. We thus explored non-injured retinal structure and function after LCT injection. LCT did not affect retinal architecture as observed by OCT (Fig. 2A; B), ERG (Fig. 2C-G) nor trigger quiescent retinal blood vessel loss (Fig. 3A). Loss of blood vessel integrity and neuronal injury result in glial activation that can be visualized by a change in the phenotype of astrocytes, microglia and Muller cells (47-49). We did not detect any changes in glial activation after a single injection of LCT (Fig. 3B; C). RPE cells form part of the outer blood-retina barrier and acts as a physical barrier to protect the retina against neovascularization (50). LCT did not modify RPE cell morphology (Fig. 3D). Our data demonstrate that a single injection of LCT preserved retinal functions, blood vessel integrity, the morphology of RPE, and did not activate astrocytes, microglia and Muller cells. All together, it indicates that LCT has no off-target effect.

Wet AMD is characterized by choroidal neovascularization that causes vision loss. Friedlander et al (14) reported the selective expression of $\alpha \mathrm{v} \beta 3$ in choroidal neovascular membranes from AMD patients while anti- $\alpha v \beta 5$ antibodies revealed only a weak immunofluorescent signal. In the rat CNV model, Nakajima et al (32) showed that 1 day after laser, the expression of fibronectin and vitronectin (ligands of $\alpha 5 \beta 1$ and $\alpha v \beta 3$ ) increased, followed by an accumulation of integrin $\alpha 5$ - and $\alpha \mathrm{v}$-positive cells around the injury area. Similarly, we demonstrated that the expression of integrin $\alpha 5$ and $\alpha \mathrm{v}$ increased at D1 and peaked at D3 in experimental mouse. LCT was thus injected intravitrealy on D0 immediately after laser injury and before the onset of integrin $\alpha 5$ - and $\alpha \mathrm{v}$-expression. A single injection of 
$500 \mu \mathrm{M}$ of LCT was able to reduce significantly lesion volume and CNV area similarly by 31.9 and $26.7 \%$ respectively. LCT reaches levels of vessel growth inhibition comparable to those obtained with anti-VEGF therapies (Fig. 5 and (51)). Similar inhibition of choroidal vessel growth were previously obtained with antagonist $\alpha 5 \beta 1(16,21)$ and $\alpha v \beta 3(19,20)$ antagonists. Surprisingly, higher inhibition of neovascular tufts was reached with EMD478761 a compound that inhibits both $\alpha v \beta 3$ and $\alpha v \beta 5$ integrins. However, this compound was effective only when sustained amounts were injected into the vitreous cavity by an intravitreal delivery device (17). LCT injected 3 days after the laser lesion was also efficient to regress neovascularization (Fig. 6) supporting the idea that a sustained delivery of LCT might help at reducing CNV.

In contrast to $\mathrm{CNV}$, both $\alpha \mathrm{v} \beta 3$ and $\alpha \mathrm{v} \beta 5$ are present on vascular cells from patients with diabetic retinopathy (12). Consistently, Takagi et al (52) demonstrated that $\alpha \mathrm{v} \beta 3$ and $\alpha \mathrm{v} \beta 5$ are expressed on neovascular tufts in the OIR mouse model. They reported that $\alpha \mathrm{v}$ subunit was gradually upregulated along with the development of neovascular vessels and peaked when the neovascularization was most prominent between P17-P19. LCT injection resulted in a reduction of pathological neovascular tufts by $48.1 \%$ whereas the revascularization of the central ischemic region was unaffected. The inhibition of pathological neovascularization was similar to the reduction observed after Aflibercept treatment (42.9\%) (Fig.7). To date, few studies have examined the effects of combined $\alpha v \beta 3, \alpha v \beta 5$ antagonism on retinal angiogenesis. Chavakis et al. (53) showed that the cyclic RGDfV peptide (antagonist of $\alpha v \beta 3$ and $\alpha v \beta 5$ ) remarkably prevented retinal neovascularization in the OIR mouse model by $57 \%$. Small molecules mimicking RGD binding motif that antagonize $\alpha v \beta 3$ and $\alpha v \beta 5$ integrins (JNJ-26076713 or SB-267268) reduced retinal neovascularization by $67 \%$ and $50 \%$ respectively $(54,55)$. However, all these molecules were only effective when administered twice daily during the 5 days of the proliferative phase of the OIR mice.

Our results, together with those reported previously, provide conclusive evidences that targeting combined $\alpha v \beta 3$, $\alpha v \beta 5$ and $\alpha 5 \beta 1$ may enhance the therapeutic effect against choroidal and retinal neovascularization.

LCT is the first $\mathrm{C}$-type lectin (interacting with $\alpha 5 \beta 1$ and $\alpha \mathrm{v}$-containing integrins) that showed efficacy in laser-induced CNV and OIR models with a single injection while other tested molecules required repeated injections. Furthermore, LCT was active with local administrations that minimize the risk of systemic effect. In conclusion, LCT appears to be highly specific of actively proliferating vascular endothelial cells with no significant effect on 
mature blood vessels, predicting a good safety profile. LCT is a strong candidate for the treatment of AMD and ischemic retinopathies. 


\section{References}

1. Friedman, D. S., O’Colmain, B. J., Muñoz, B., Tomany, S. C., McCarty, C., de Jong, P. T. V. M., Nemesure, B., Mitchell, P., and Kempen, J., Eye Diseases Prevalence Research Group. (2004) Prevalence of age-related macular degeneration in the United States. Arch. Ophthalmol. Chic. Ill 1960 122, 564-572

2. Kempen, J. H., O’Colmain, B. J., Leske, M. C., Haffner, S. M., Klein, R., Moss, S. E., Taylor, H. R., and Hamman, R. F., Eye Diseases Prevalence Research Group. (2004) The prevalence of diabetic retinopathy among adults in the United States. Arch. Ophthalmol. Chic. Ill 1960 122, 552-563

3. Klein, R., Peto, T., Bird, A., and Vannewkirk, M. R. (2004) The epidemiology of agerelated macular degeneration. Am. J. Ophthalmol. 137, 486-495

4. D'Amore, P. A. (1994) Mechanisms of retinal and choroidal neovascularization. Invest. Ophthalmol. Vis. Sci. 35, 3974-3979

5. Brown, D. M., Kaiser, P. K., Michels, M., Soubrane, G., Heier, J. S., Kim, R. Y., Sy, J. P., and Schneider, S., ANCHOR Study Group. (2006) Ranibizumab versus verteporfin for neovascular age-related macular degeneration. N. Engl. J. Med. 355, 1432-1444

6. Rosenfeld, P. J., Brown, D. M., Heier, J. S., Boyer, D. S., Kaiser, P. K., Chung, C. Y., and Kim, R. Y., MARINA Study Group. (2006) Ranibizumab for neovascular age-related macular degeneration. N. Engl. J. Med. 355, 1419-1431

7. Forooghian, F., Cukras, C., Meyerle, C. B., Chew, E. Y., and Wong, W. T. (2009) Tachyphylaxis Following Intravitreal Bevacizumab for Exudative Age-Related Macular Degeneration. Retina Phila. Pa 29, 723-731

8. Eghøj, M. S. and Sørensen, T. L. (2012) Tachyphylaxis during treatment of exudative age-related macular degeneration with ranibizumab. Br. J. Ophthalmol. 96, 21-23

9. Martinez-Zapata, M. J., Martí-Carvajal, A. J., Solà, I., Pijoán, J. I., Buil-Calvo, J. A., Cordero, J. A., and Evans, J. R. (2014) Anti-vascular endothelial growth factor for proliferative diabetic retinopathy. Cochrane Database Syst. Rev. 11, CD008721

10. Bishop, P. N. (2015) The role of extracellular matrix in retinal vascular development and preretinal neovascularization. Exp. Eye Res. 133, 30-36

11. Brooks, P. C., Montgomery, A. M., Rosenfeld, M., Reisfeld, R. A., Hu, T., Klier, G., and Cheresh, D. A. (1994) Integrin alpha v beta 3 antagonists promote tumor regression by inducing apoptosis of angiogenic blood vessels. Cell 79, 1157-1164

12. Friedlander, M., Brooks, P. C., Shaffer, R. W., Kincaid, C. M., Varner, J. A., and Cheresh, D. A. (1995) Definition of two angiogenic pathways by distinct alpha $\mathrm{v}$ integrins. Science 270, 1500-1502

13. Robbins, S. G., Brem, R. B., Wilson, D. J., O’Rourke, L. M., Robertson, J. E., Westra, I., Planck, S. R., and Rosenbaum, J. T. (1994) Immunolocalization of integrins in proliferative retinal membranes. Invest. Ophthalmol. Vis. Sci. 35, 3475-3485 
14. Friedlander, M., Theesfeld, C. L., Sugita, M., Fruttiger, M., Thomas, M. A., Chang, S., and Cheresh, D. A. (1996) Involvement of integrins alpha $v$ beta 3 and alpha $v$ beta 5 in ocular neovascular diseases. Proc. Natl. Acad. Sci. U. S. A. 93, 9764-9769

15. Goodman, S. L. and Picard, M. (2012) Integrins as therapeutic targets. Trends Pharmacol. Sci. 33, 405-412

16. Umeda, N., Kachi, S., Akiyama, H., Zahn, G., Vossmeyer, D., Stragies, R., and Campochiaro, P. A. (2006) Suppression and Regression of Choroidal Neovascularization by Systemic Administration of an $\alpha 5 \beta 1$ Integrin Antagonist. Mol. Pharmacol. 69, 1820-1828

17. Fu, Y., Ponce, M. L., Thill, M., Yuan, P., Wang, N. S., and Csaky, K. G. (2007) Angiogenesis inhibition and choroidal neovascularization suppression by sustained delivery of an integrin antagonist, EMD478761. Invest. Ophthalmol. Vis. Sci. 48, 5184-5190

18. Zahn, G., Vossmeyer, D., Stragies, R., Wills, M., Wong, C. G., Löffler, K. U., Adamis, A. P., and Knolle, J. (2009) Preclinical evaluation of the novel small-molecule integrin alpha5beta1 inhibitor JSM6427 in monkey and rabbit models of choroidal neovascularization. Arch. Ophthalmol. Chic. Ill 1960 127, 1329-1335

19. Honda, S., Nagai, T., and Negi, A. (2009) Anti-angiogenic effects of non-peptide integrin alphavbeta3 specific antagonist on laser-induced choroidal neovascularization in mice. Graefes Arch. Clin. Exp. Ophthalmol. Albrecht Von Graefes Arch. Für Klin. Exp. Ophthalmol. 247, 515-522

20. Salehi-Had, H., Roh, M. I., Giani, A., Hisatomi, T., Nakao, S., Kim, I. K., Gragoudas, E. S., Vavvas, D., Guccione, S., and Miller, J. W. (2011) Utilizing Targeted Gene Therapy with Nanoparticles Binding Alpha v Beta 3 for Imaging and Treating Choroidal Neovascularization. PLoS One 6, e18864

21. Wang, W., Wang, F., Lu, F., Xu, S., Hu, W., Huang, J., Gu, Q., and Sun, X. (2011) The antiangiogenic effects of integrin alpha5beta1 inhibitor (ATN-161) in vitro and in vivo. Invest. Ophthalmol. Vis. Sci. 52, 7213-7220

22. Sarray, S., Srairi, N., Hatmi, M., Luis, J., Louzir, H., Regaya, I., Slema, H., Marvaldi, J., El Ayeb, M., and Marrakchi, N. (2003) Lebecetin, a potent antiplatelet C-type lectin from Macrovipera lebetina venom. Biochim. Biophys. Acta 1651, 30-40

23. Sarray, S., Delamarre, E., Marvaldi, J., El Ayeb, M., Marrakchi, N., and Luis, J. (2007) Lebectin and lebecetin, two C-type lectins from snake venom, inhibit alpha5beta1 and alphaV-containing integrins. Matrix Biol. J. Int. Soc. Matrix Biol. 26, 306-313

24. Pilorget, A., Conesa, M., Sarray, S., Michaud-Levesque, J., Daoud, S., Kim, K. S., Demeule, M., Marvaldi, J., El Ayeb, M., Marrakchi, N., Béliveau, R., and Luis, J. (2007) Lebectin, a Macrovipera lebetina venom-derived C-type lectin, inhibits angiogenesis both in vitro and in vivo. J. Cell. Physiol. 211, 307-315

25. Lavalette, S., Raoul, W., Houssier, M., Camelo, S., Levy, O., Calippe, B., Jonet, L., Behar-Cohen, F., Chemtob, S., Guillonneau, X., Combadière, C., and Sennlaub, F. (2011) Interleukin-1 $\beta$ Inhibition Prevents Choroidal Neovascularization and Does Not Exacerbate Photoreceptor Degeneration. Am. J. Pathol. 178, 2416-2423 
26. Shao, Z., Friedlander, M., Hurst, C. G., Cui, Z., Pei, D. T., Evans, L. P., Juan, A. M., Tahir, H., Duhamel, F., Chen, J., Sapieha, P., Chemtob, S., Joyal, J.-S., and Smith, L. E. H. (2013) Choroid Sprouting Assay: An Ex Vivo Model of Microvascular Angiogenesis. PLoS One 8, e69552

27. Dominguez, E., Raoul, W., Calippe, B., Sahel, J.-A., Guillonneau, X., Paques, M., and Sennlaub, F. (2015) Experimental Branch Retinal Vein Occlusion Induces Upstream Pericyte Loss and Vascular Destabilization. PLoS One 10, e0132644

28. Schindelin, J., Arganda-Carreras, I., Frise, E., Kaynig, V., Longair, M., Pietzsch, T., Preibisch, S., Rueden, C., Saalfeld, S., Schmid, B., Tinevez, J.-Y., White, D. J., Hartenstein, V., Eliceiri, K., Tomancak, P., and Cardona, A. (2012) Fiji: an open-source platform for biological-image analysis. Nat. Methods 9, 676-682

29. Berger, A., Cavallero, S., Dominguez, E., Barbe, P., Simonutti, M., Sahel, J.-A., Sennlaub, F., Raoul, W., Paques, M., and Bemelmans, A.-P. (2014) Spectral-Domain Optical Coherence Tomography of the Rodent Eye: Highlighting Layers of the Outer Retina Using Signal Averaging and Comparison with Histology. PLoS One 9, e96494

30. Connor, K. M., Krah, N. M., Dennison, R. J., Aderman, C. M., Chen, J., Guerin, K. I., Sapieha, P., Stahl, A., Willett, K. L., and Smith, L. E. H. (2009) Quantification of oxygeninduced retinopathy in the mouse: a model of vessel loss, vessel regrowth and pathological angiogenesis. Nat. Protoc. 4, 1565-1573

31. Remtulla, S. and Hallett, P. E. (1985) A schematic eye for the mouse, and comparisons with the rat. Vision Res. 25, 21-31

32. Nakajima, T., Hirata, M., Shearer, T. R., and Azuma, M. (2014) Mechanism for laserinduced neovascularization in rat choroid: Accumulation of integrin $\alpha$ chain-positive cells and their ligands. Mol. Vis. 20, 864-871

33. Launay, P.-S., Reboussin, E., Liang, H., Kessal, K., Godefroy, D., Rostene, W., Sahel, J.-A., Baudouin, C., Melik Parsadaniantz, S., and Reaux Le Goazigo, A. (2016) Ocular inflammation induces trigeminal pain, peripheral and central neuroinflammatory mechanisms. Neurobiol. Dis. 88, 16-28

34. Tsujino, H., Kondo, E., Fukuoka, T., Dai, Y., Tokunaga, A., Miki, K., Yonenobu, K., Ochi, T., and Noguchi, K. (2000) Activating transcription factor 3 (ATF3) induction by axotomy in sensory and motoneurons: A novel neuronal marker of nerve injury. Mol. Cell. Neurosci. 15, 170-182

35. Kovach, J. L., Schwartz, S. G., Flynn, H. W., and Scott, I. U. (2012) Anti-VEGF Treatment Strategies for Wet AMD. J. Ophthalmol. 2012

36. Papadopoulos, N., Martin, J., Ruan, Q., Rafique, A., Rosconi, M. P., Shi, E., Pyles, E. A., Yancopoulos, G. D., Stahl, N., and Wiegand, S. J. (2012) Binding and neutralization of vascular endothelial growth factor (VEGF) and related ligands by VEGF Trap, ranibizumab and bevacizumab. Angiogenesis 15, 171-185

37. Lambert, V., Lecomte, J., Hansen, S., Blacher, S., Gonzalez, M.-L. A., Struman, I., Sounni, N. E., Rozet, E., de Tullio, P., Foidart, J. M., Rakic, J.-M., and Noel, A. (2013) Laser- 
induced choroidal neovascularization model to study age-related macular degeneration in mice. Nat. Protoc. 8, 2197-2211

38. Smith, L. E., Wesolowski, E., McLellan, A., Kostyk, S. K., D’Amato, R., Sullivan, R., and D'Amore, P. A. (1994) Oxygen-induced retinopathy in the mouse. Invest. Ophthalmol. Vis. Sci. 35, 101-111

39. Stahl, A., Connor, K. M., Sapieha, P., Willett, K. L., Krah, N. M., Dennison, R. J., Chen, J., Guerin, K. I., and Smith, L. E. H. (2009) Computer-aided quantification of retinal neovascularization. Angiogenesis 12, 297-301

40. Tokunaga, C. C., Mitton, K. P., Dailey, W., Massoll, C., Roumayah, K., Guzman, E., Tarabishy, N., Cheng, M., and Drenser, K. A. (2014) Effects of anti-VEGF treatment on the recovery of the developing retina following oxygen-induced retinopathy. Invest. Ophthalmol. Vis. Sci. 55, 1884-1892

41. Alghisi, G. C. and Rüegg, C. (2006) Vascular integrins in tumor angiogenesis: mediators and therapeutic targets. Endothel. J. Endothel. Cell Res. 13, 113-135

42. Legler, D. F., Doucey, M. A., Cerottini, J. C., Bron, C., and Luescher, I. F. (2001) Selective inhibition of CTL activation by a dipalmitoyl-phospholipid that prevents the recruitment of signaling molecules to lipid rafts. FASEB J. Off. Publ. Fed. Am. Soc. Exp. Biol. 15, 1601-1603

43. Reynolds, A. R., Hart, I. R., Watson, A. R., Welti, J. C., Silva, R. G., Robinson, S. D., Da Violante, G., Gourlaouen, M., Salih, M., Jones, M. C., Jones, D. T., Saunders, G., Kostourou, V., Perron-Sierra, F., Norman, J. C., Tucker, G. C., and Hodivala-Dilke, K. M. (2009) Stimulation of tumor growth and angiogenesis by low concentrations of RGD-mimetic integrin inhibitors. Nat. Med. 15, 392-400

44. Kim, S., Bakre, M., Yin, H., and Varner, J. A. (2002) Inhibition of endothelial cell survival and angiogenesis by protein kinase A. J. Clin. Invest. 110, 933-941

45. Kim, S., Bell, K., Mousa, S. A., and Varner, J. A. (2000) Regulation of Angiogenesis in Vivo by Ligation of Integrin $\alpha 5 \beta 1$ with the Central Cell-Binding Domain of Fibronectin. Am. J. Pathol. 156, 1345-1362

46. Bader, B. L., Rayburn, H., Crowley, D., and Hynes, R. O. (1998) Extensive Vasculogenesis, Angiogenesis, and Organogenesis Precede Lethality in Mice Lacking All $\alpha \mathrm{v}$ Integrins. Cell 95, 507-519

47. Buffo, A., Rolando, C., and Ceruti, S. (2010) Astrocytes in the damaged brain: molecular and cellular insights into their reactive response and healing potential. Biochem. Pharmacol. 79, 77-89

48. Wang, M., Ma, W., Zhao, L., Fariss, R. N., and Wong, W. T. (2011) Adaptive Müller cell responses to microglial activation mediate neuroprotection and coordinate inflammation in the retina. J. Neuroinflammation 8, 173

49. Langmann, T. (2007) Microglia activation in retinal degeneration. J. Leukoc. Biol. 81, $1345-1351$ 
50. Strauss, O. (2005) The retinal pigment epithelium in visual function. Physiol. Rev. 85, $845-881$

51. Huang, H., Shen, J., and Vinores, S. A. (2011) Blockade of VEGFR1 and 2 Suppresses Pathological Angiogenesis and Vascular Leakage in the Eye. PLoS One 6, e21411

52. Takagi, H., Suzuma, K., Otani, A., Oh, H., Koyama, S., Ohashi, H., Watanabe, D., Ojima, T., Suganami, E., and Honda, Y. (2002) Role of vitronectin receptor-type integrins and osteopontin in ischemia-induced retinal neovascularization. Jpn. J. Ophthalmol. 46, 270278

53. Chavakis, E., Riecke, B., Lin, J., Linn, T., Bretzel, R. G., Preissner, K. T., Brownlee, M., and Hammes, H.-P. (2002) Kinetics of integrin expression in the mouse model of proliferative retinopathy and success of secondary intervention with cyclic RGD peptides. Diabetologia 45, 262-267

54. Wilkinson-Berka, J. L., Jones, D., Taylor, G., Jaworski, K., Kelly, D. J., Ludbrook, S. B., Willette, R. N., Kumar, S., and Gilbert, R. E. (2006) SB-267268, a nonpeptidic antagonist of alpha(v)beta3 and alpha(v)beta5 integrins, reduces angiogenesis and VEGF expression in a mouse model of retinopathy of prematurity. Invest. Ophthalmol. Vis. Sci. 47, 1600-1605

55. Santulli, R. J., Kinney, W. A., Ghosh, S., Decorte, B. L., Liu, L., Tuman, R. W. A., Zhou, Z., Huebert, N., Bursell, S. E., Clermont, A. C., Grant, M. B., Shaw, L. C., Mousa, S. A., Galemmo, R. A., Johnson, D. L., Maryanoff, B. E., and Damiano, B. P. (2008) Studies with an orally bioavailable alpha $\mathrm{V}$ integrin antagonist in animal models of ocular vasculopathy: retinal neovascularization in mice and retinal vascular permeability in diabetic rats. J. Pharmacol. Exp. Ther. 324, 894-901 


\section{Author Contributions.}

F. Montassar, F. Sennlaub, N. Marrakchi, E. Messadi, X. Guillonneau designed research. F. Montassar, M. Darche, A. Blaizot, A. Augustin, J-B. Conart, A. Millet, A. Réaux-Le-Goazigo performed research. A. Réaux-Le-Goazigo contributed new reagents or analytic tools. F. Montassar, F. Sennlaub, N. Marrakchi, E. Messadi, X. Guillonneau analyzed research. F. Montassar, F. Sennlaub, M. Elayeb, J-A. Sahel, N. Marrakchi, E. Messadi, X. Guillonneau analyzed data. F. Montassar, F. Sennlaub, E. Messadi, X. Guillonneau wrote the paper.

\section{Acknowledgements}

This work was supported by grants from INSERM, Labex Lifesenses, Carnot, PHC-Utique Program (the Ministère de l'enseignement Supérieur et de la Recherche Scientifique de Tunisie and the Ministère des Affaires Etrangères de France) and the Institute Pasteur International Network (RIIP). The authors declare no conflicts of interest. 


\section{Figure legends}

Figure 1: LCT inhibits vascular sprouting from aortic and choroidal explants.

(A, C) Representative microphotographs of aortic and choroidal endothelial sprouts. (B) Measurement of vascular sprouting from aortic rings from P4 Lewis rat pups in control and LCT (30 nM, $300 \mathrm{nM}, 1.5 \mu \mathrm{M})$ groups, ( $n=4$ per group, ${ }^{*} p 0.05$; one-way ANOVA followed by Dunnett's post-test; CTL as control, representative of 3 independent experiments). (D) Measurement of vascular sprouting from choroidal explants from 3-weeks-old C57BL/6JRj male mice in control and LCT $(1.5 \mu \mathrm{M}, 5 \mu \mathrm{M}, 15 \mu \mathrm{M})$ groups, $\left(n \geq 5\right.$ per group, ${ }^{*} p<0.05$; one-way ANOVA followed by Dunnett's post-test; CTL as control, representative of 3 independent experiments). Scale bars in A and $\mathrm{C}=200 \mu \mathrm{m}$.

Figure 2: LCT intravitreal injection does not alter visual function

(A) Representative SD-OCT image of retina at D7 post-intravitreal injection of $1 \mu \mathrm{l}$ of PBS or LCT $(500 \mu \mathrm{M})$. (B) Quantification of the thickness of the entire retina, ONL, INL and OS at $500 \mu \mathrm{m}$ of the optic nerve, $\mathrm{n}=4$ eyes per group. (C) Representative electroretinogram traces from scotopic ERG recording of CTL, PBS and LCT groups at $0.3 \mathrm{cds} / \mathrm{m}^{2}$. (D) Scotopic recorded a- and b-waves amplitudes at different stimulus intensities $\left(0.003 \mathrm{~cd} . \mathrm{s} / \mathrm{m}^{2} ; 0.03 \mathrm{~cd} . \mathrm{s} /\right.$ $\mathrm{m}^{2} ; 0.3$ cd.s $/ \mathrm{m}^{2} ; 3$ cd.s $/ \mathrm{m}^{2} ; 10$ cd.s/ $\mathrm{m}^{2}$ ). (E) Photopic response amplitudes at flash intensity of $10 \mathrm{~cd} . \mathrm{s} / \mathrm{m}^{2}$ of light adapted animals. (F) Representative electroretinogram traces from ERG flickers recorded at flash frequencies of $10 \mathrm{~Hz}$ at $1 \mathrm{~cd} . \mathrm{s} / \mathrm{m}^{2}$ intensity. (G) Flicker response amplitudes recorded at flash frequencies of 10 and $20 \mathrm{~Hz}, \mathrm{n}=10$ eyes per group for each ERG recording. IPL: Inner plexiform layer, INL: Inner nuclear layer, ONL: Outer nuclear layer, OLM: Outer limiting membrane. Scale bar in $\mathrm{A}=100 \mu \mathrm{m}$.

Figure 3: LCT intravitreal injection does not alter vascular integrity

(A) Microphotographs of retinal flatmounts from C57BL/6JRj mice immunostained with FITC-coupled BS-1 lectin (green), collagen-IV (red), and (B) GFAP (green) 7 days after intravitreal injections of PBS $(1 \mu \mathrm{l})$ or LCT $(1 \mu \mathrm{l}, 500 \mu \mathrm{M})$. (C) Microphotographs of retinal flatmounts from $\mathrm{CX} 3 \mathrm{CR} 1^{+} / \mathrm{GFP}$ mice immunostained with collagen-IV (red) and Iba1 (green) antibodies in the inner and outer plexus 7 days after intravitreal injections of PBS ( $1 \mu \mathrm{l})$ or LCT $(1 \mu \mathrm{l}, 500 \mu \mathrm{M})$. (D) Choroidal flatmounts from C57BL/6JRj mice co-immunostained with TRITC-coupled phalloidin (red) and DAPI (blue) at D7 after intravitreal injections of 
PBS $(1 \mu \mathrm{l})$ or LCT $(1 \mu \mathrm{l}, 500 \mu \mathrm{M})$. Scale bars in A, B and C $=50 \mu \mathrm{m}$; in $\mathrm{D}=100 \mu \mathrm{m}$.

Figure 4: LCT binds to CNV lesions

(A) Quantitative RT-PCR of integrin subunit mRNA ( $\alpha \mathrm{v}, \alpha 5, \beta 3, \beta 5)$ normalized with GADPH mRNA of C57BL/6JRj choroids collected at days $0,1,3$, and 7, after laserinduced choroidal lesions, $n=8$ eyes per group (B) Microphotographs of laser-induced CNV lesions at D7 on choroidal flatmounts after intravitreal injections on D4 of bovine serum albumin (647-BSA), LCT (647-LCT) or Aflibercept (647-Aflibercept) covalently conjugated to an Alexa Fluor 647 dye . All choroids were co-stained with CD102 antibody (green). Scale bars in $\mathrm{B}-\mathrm{E}=50 \mu \mathrm{m}$.

Figure 5: LCT inhibits laser-induced choroidal neovascularization

(A) Representative SD-OCT images of choroidal lesion and quantification of lesion volume 7 days after laser and intravitreal injections of PBS $(1 \mu \mathrm{l})$, LCT $(1 \mu 1,500 \mu \mathrm{M})$ and Aflibercept $(1 \mu \mathrm{l}, 25 \mu \mathrm{M})$. (n=26, 35 and 16 respectively laser impacts per group. ${ }^{*} p<0.05$; one-way ANOVA followed by Bonferroni post-test; representative of 2 independent experiments) Lesion volume is extrapolated using following formula $\left(4 / 3 \pi^{*} \mathrm{a}^{*} \mathrm{~b}^{2}\right) / 2, a$ is the polar radius (vertical axis) and $b$ is the equator radius (horizontal axis). (B) Microphotographs of CNV lesion on choroidal flatmounts of PBS $(1 \mu \mathrm{l})$, LCT $(1 \mu 1,500 \mu \mathrm{M})$ and Aflibercept $(1 \mu 1,25$ $\mu \mathrm{M}$ ) stained with CD102 (green), Iba1 (red) and DAPI (blue). (C) Quantification of CNV (CD102-positive area) 7 days after laser and intravitreal injections of PBS (1 $\mu 1)$, LCT (1 $\mu$, $500 \mu \mathrm{M})$ and Aflibercept $(1 \mu 1,25 \mu \mathrm{M})(\mathrm{n}=29,32$ and 30 respectively laser impacts per group. $* p<0.05$; one-way ANOVA followed by Bonferroni post-test; representative of 2 independent experiments). (D) Quantification of Iba1 positive cells per impact 7 days after laser and intravitreal injections of PBS $(1 \mu \mathrm{l})$, LCT $(1 \mu \mathrm{l}, 500 \mu \mathrm{M})$ and Aflibercept $(1 \mu 1,25 \mu \mathrm{M})(\mathrm{n}=29$, 32 and 30 respectively laser impacts per group. ${ }^{*} p<0.05$; one-way ANOVA followed by Bonferroni post-test; representative of 2 independent experiments). (E) Quantitative RT-PCR of integrin subunit mRNA ( $\alpha \mathrm{v}, \alpha 5, \beta 3, \beta 5)$ normalized with GADPH mRNA of C57BL/6JRj choroids at D3. Mice were treated with a single intravitreal injection of PBS (1 1 l) or LCT (1 $\mu 1,500 \mu \mathrm{M})$ at $\mathrm{D} 2, \mathrm{n}=5$ eyes per group. INL: inner nuclear layer, ONL: outer nuclear layer, Scale bars in $\mathrm{A}=50 \mu \mathrm{m}$; in $\mathrm{B}=100 \mu \mathrm{m}$. 
Figure 6: LCT intravitreal injection at D3 inhibits choroidal neovascularization

(A) Microphotographs of CNV lesions stained with CD102 (green), and DAPI (blue) 7 days after laser (D0) and intravitreal injections (D3) of PBS (1 $\mu \mathrm{l})$, LCT $(1 \mu \mathrm{l}, 500 \mu \mathrm{M})$. (B) Quantification of CNV (CD102-positive area) on PBS and LCT choroidal flatmounts at D7 ( $\mathrm{n}=27$ and 28 laser impacts per group. ${ }^{*} p<0.05$; Mann-Whitney $\mathrm{U}$ test; representative of 2 independent experiments), Scale bar in $\mathrm{A}=100 \mu \mathrm{m}$.

Figure 7: LCT inhibits retinal neovascularization in the Oxygen Induced Retinopathy (OIR) model.

(A) Microphotographs of FITC-coupled BS-1 lectin-stained retina (green) of P17-OIR C57BL/6JRj mice that received an intravitreal injection at P14 of LCT (647-LCT) covalently conjugated to an Alexa Fluor 647 dye (red). (B) Microphotographs of FITC-coupled BS-1 lectin-stained retina, of P17-OIR C57BL/6JRj mice, after intravitreal injection at P12 of PBS $(1 \mu \mathrm{l})$, LCT $(1 \mu 1,500 \mu \mathrm{M})$ or Aflibercept $(1 \mu 1,25 \mu \mathrm{M})$. Neovascularization (NV) and vasoobliteration (VO) areas were highlighted in white and red respectively. (C) Representative confocal microscopy photographs of neovascularization in BS-1 lectin-stained control and treated retinas. (D) Quantification of vaso-obliteration and (E) neovascularization (BS-1 lectin positive-area) in P17 control and treated retinal flatmounts, $(n=35,32$ and 16 OIR-retina respectively. ${ }^{*} p<0.05$; one-way ANOVA followed by Bonferroni post-test; representative of 2 independent experiments). Scale bars in $\mathrm{A}=30 \mu \mathrm{M}$; in $\mathrm{B}=80 \mathrm{~mm}$; and in $\mathrm{C}=100 \mu \mathrm{m}$. 

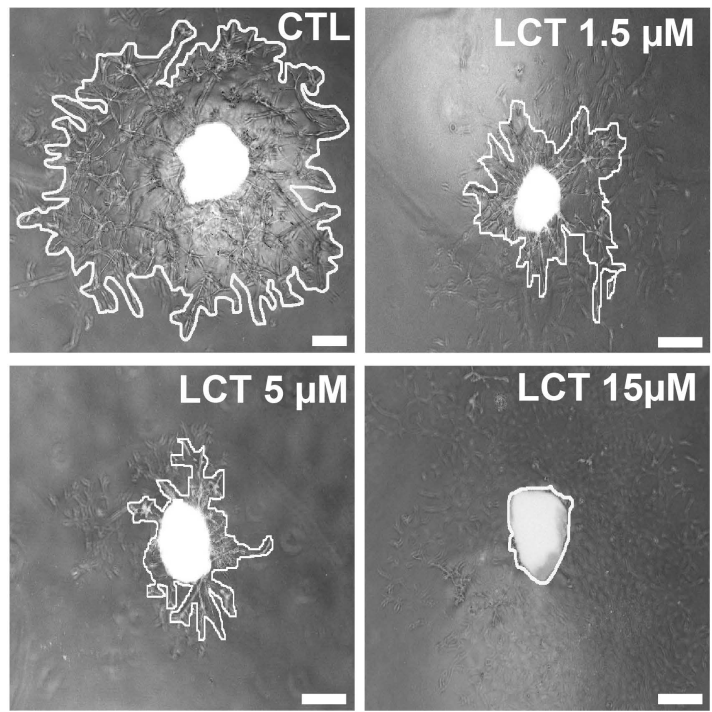
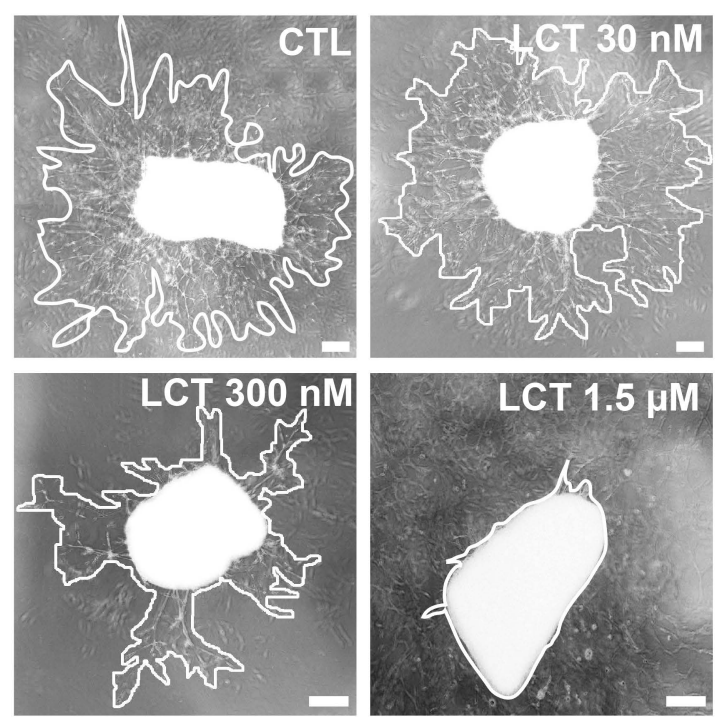

C
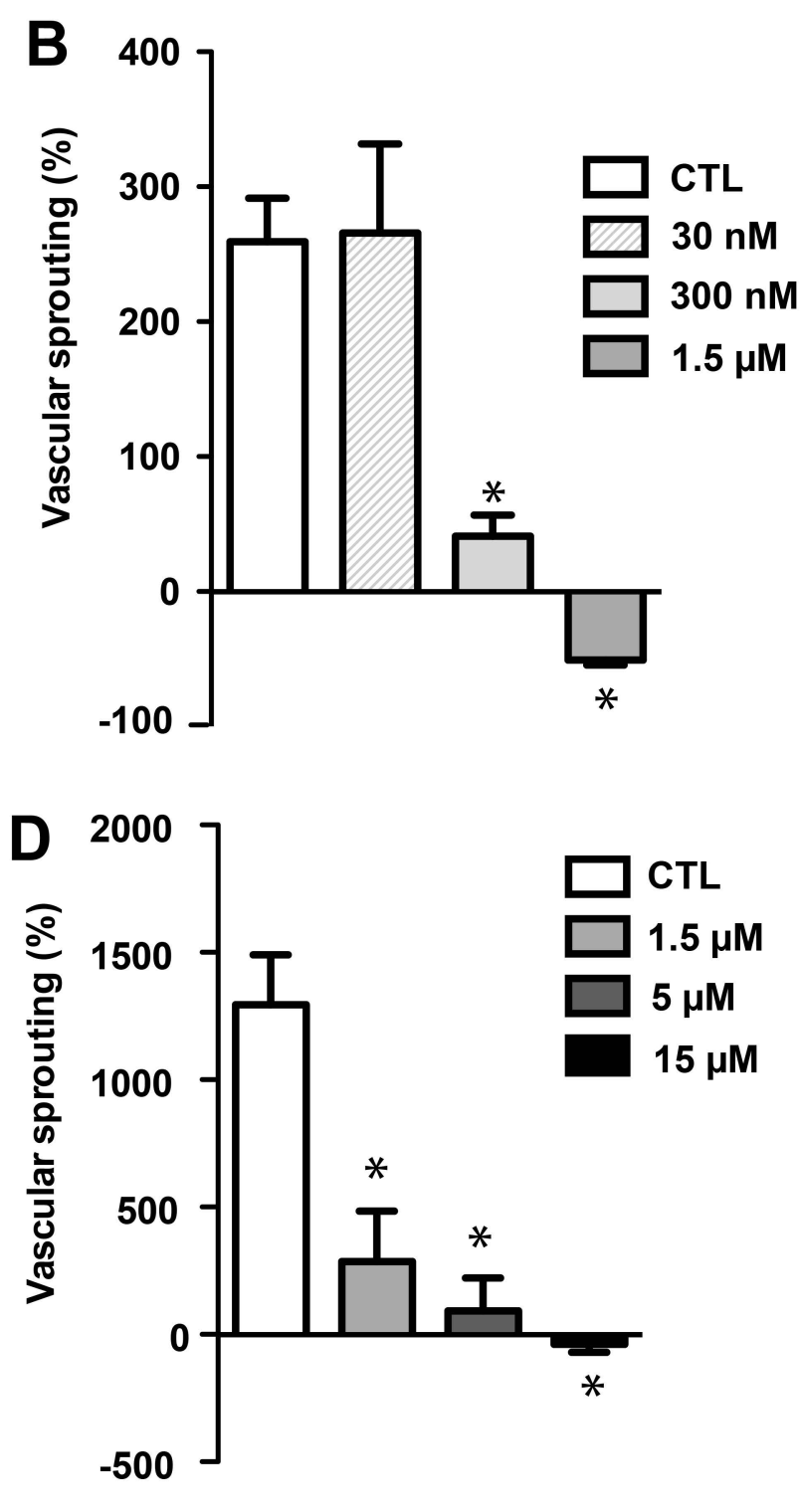

Figure 1 

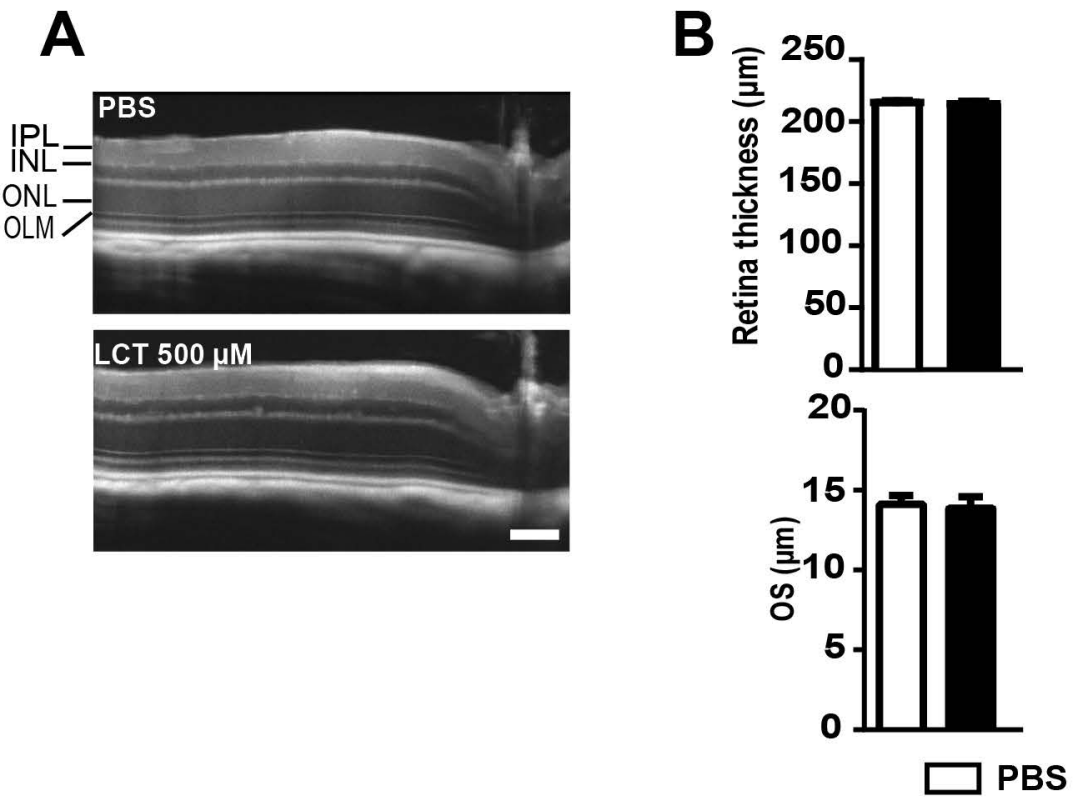
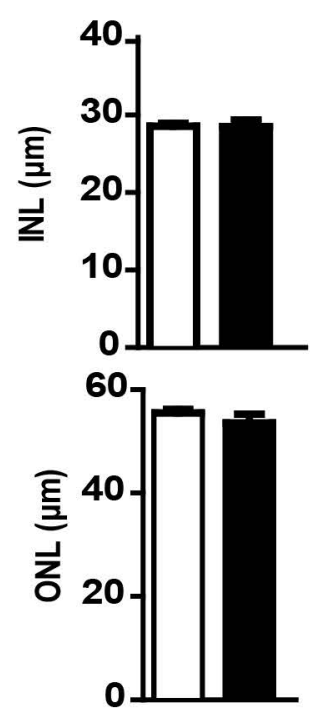

LCT $500 \mu \mathrm{M}$

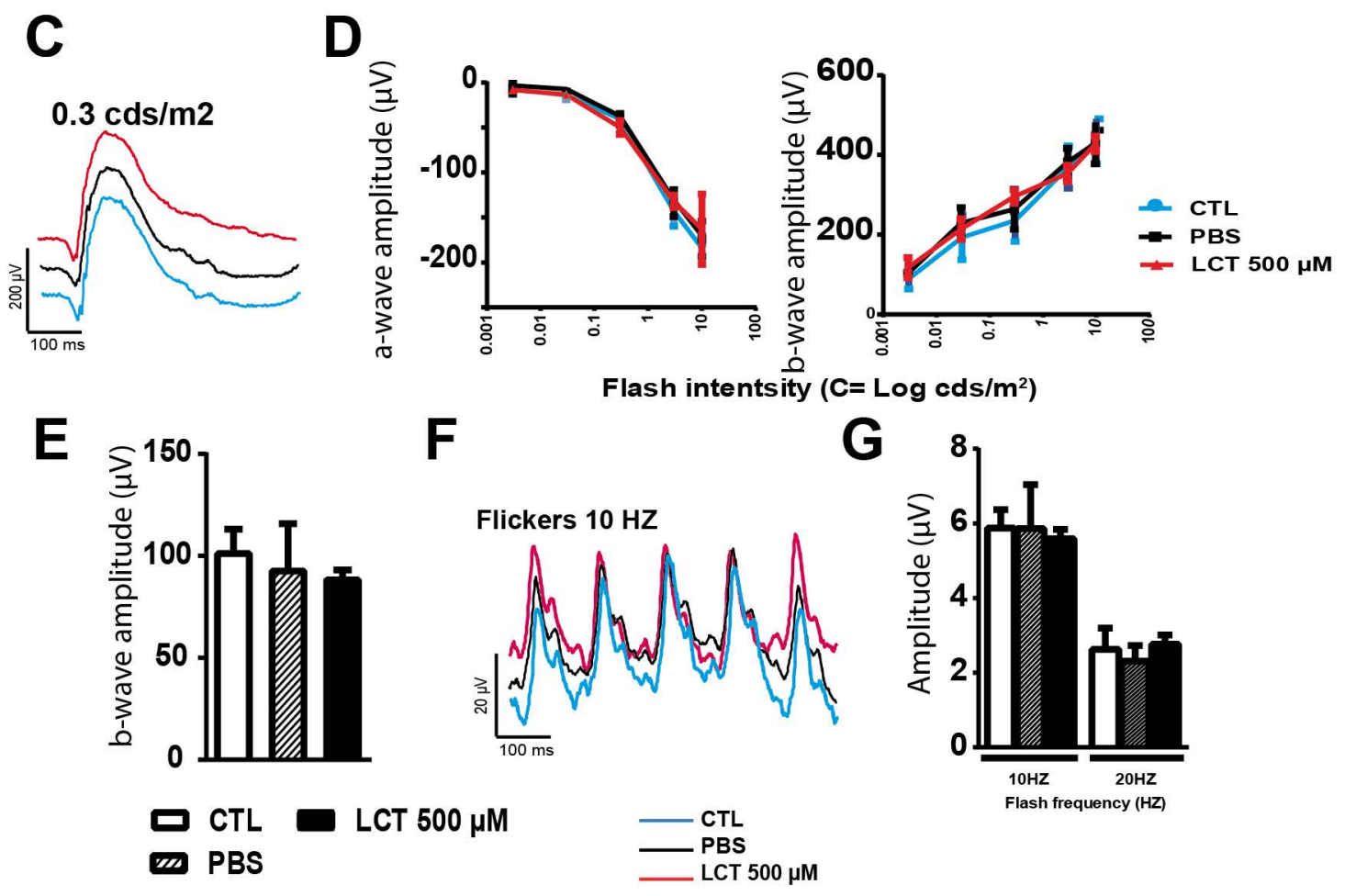

Figure 2 
A PBS

\section{PBS}
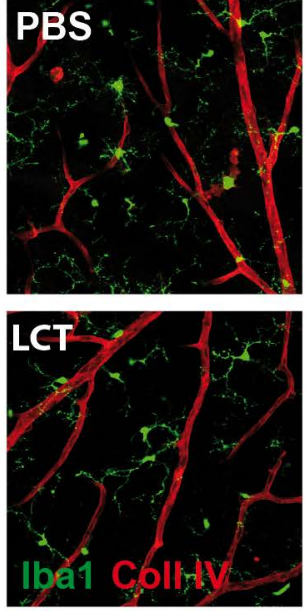

$B$ PBS
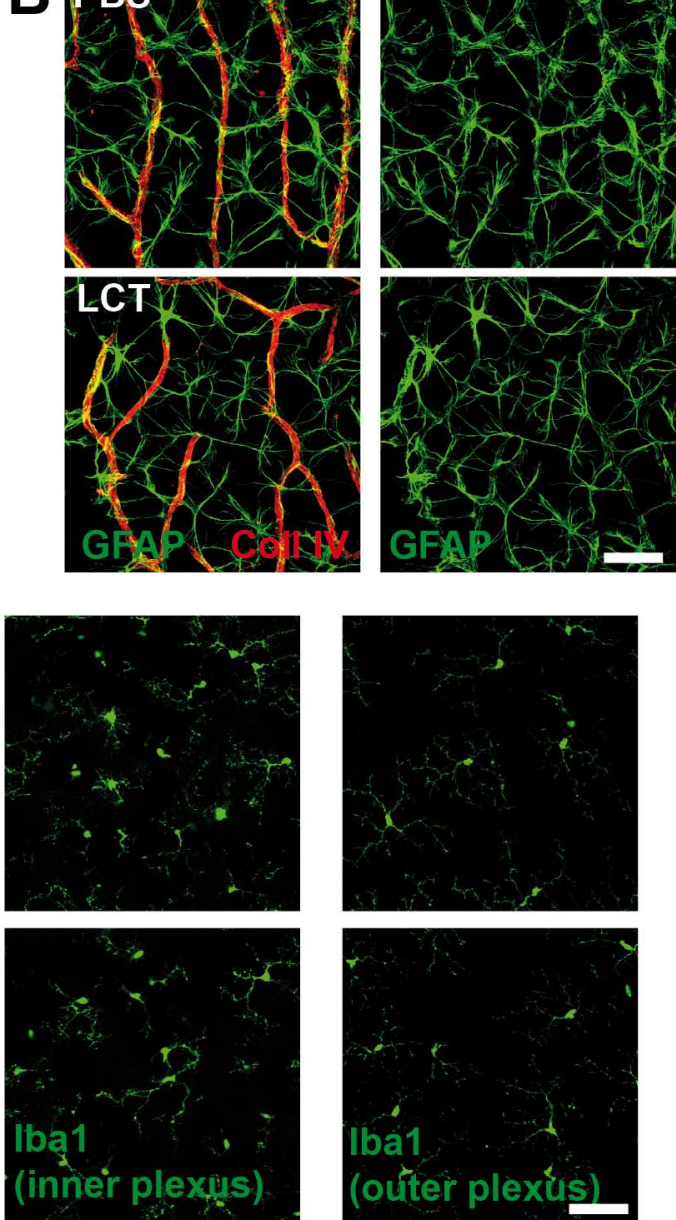
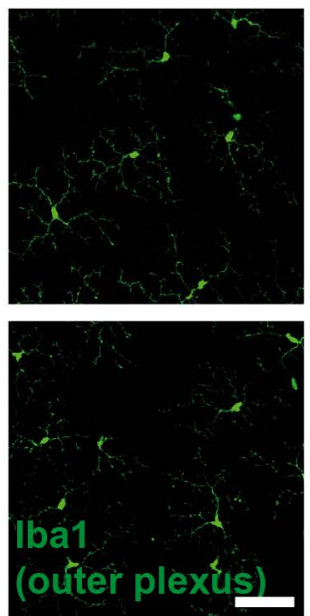

D
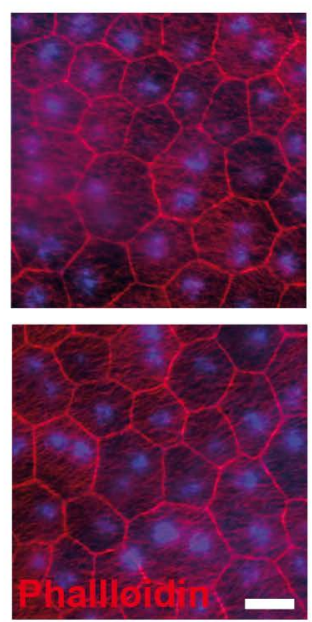

Figure 3 
A
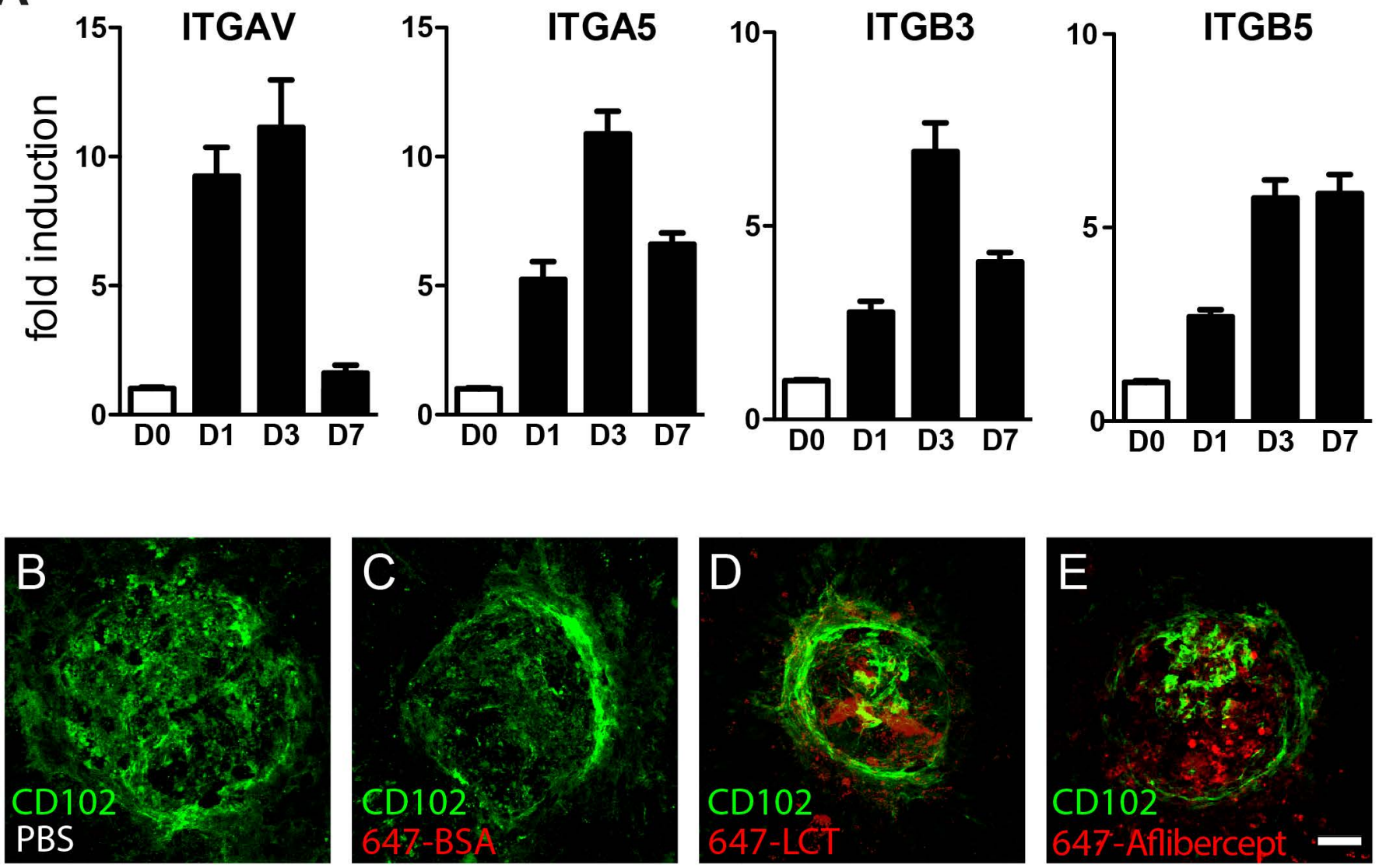

Figure 4 
A
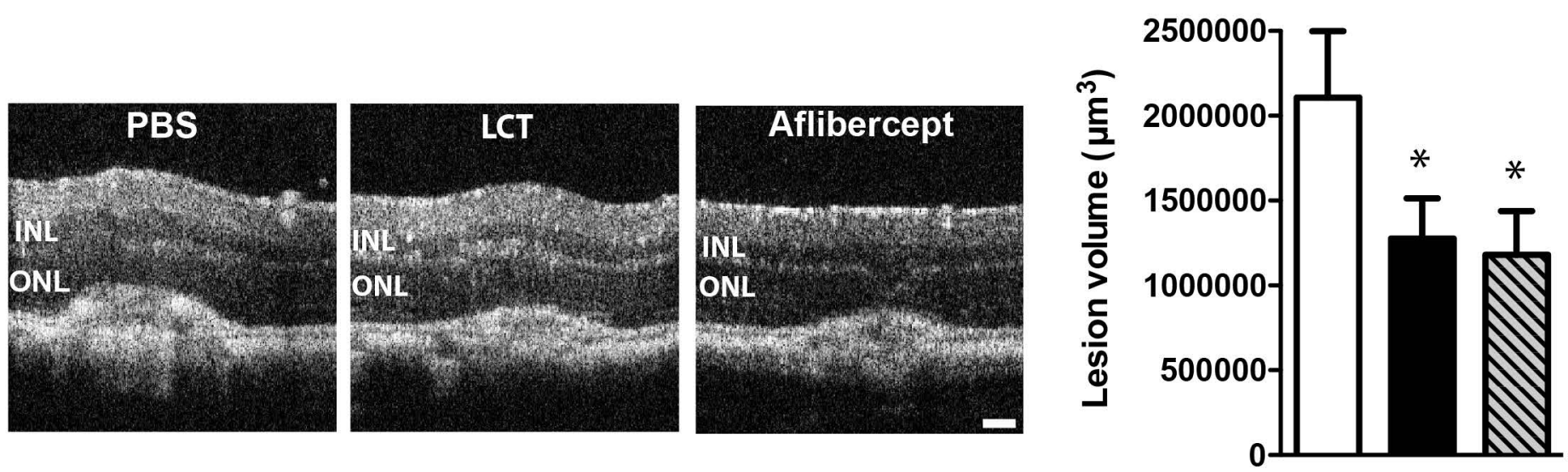

B
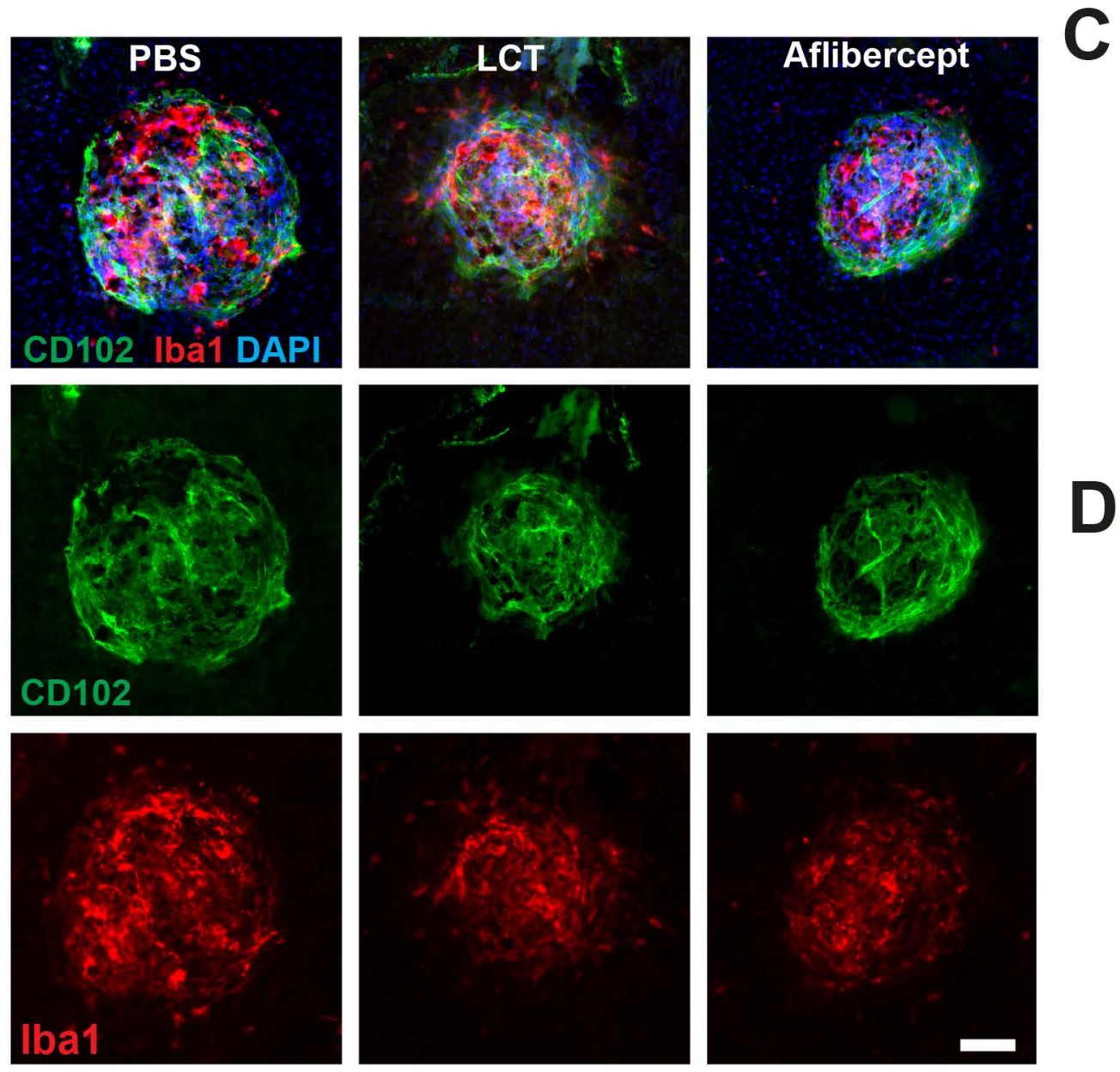

D

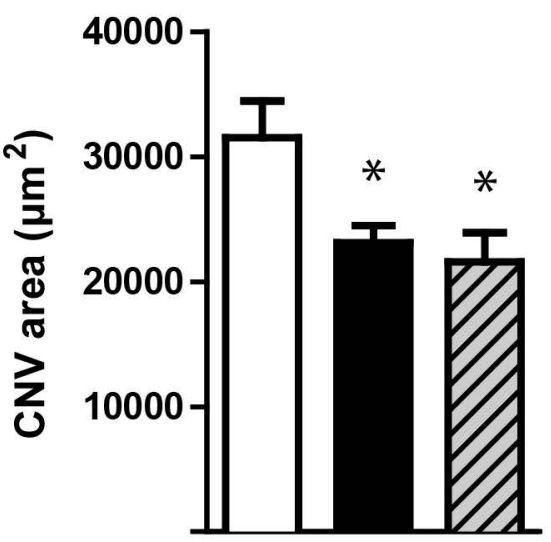

E
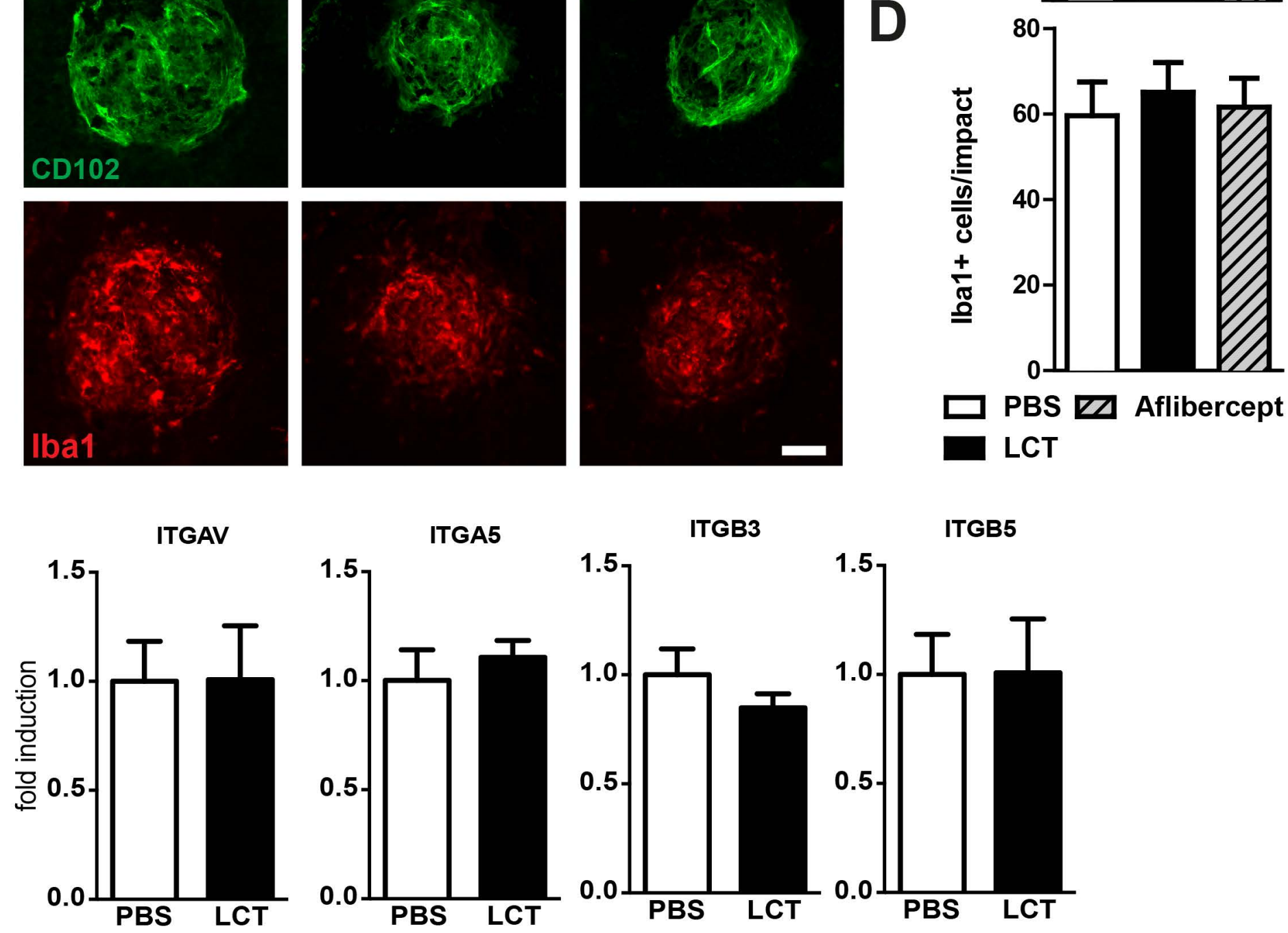

ITGB5

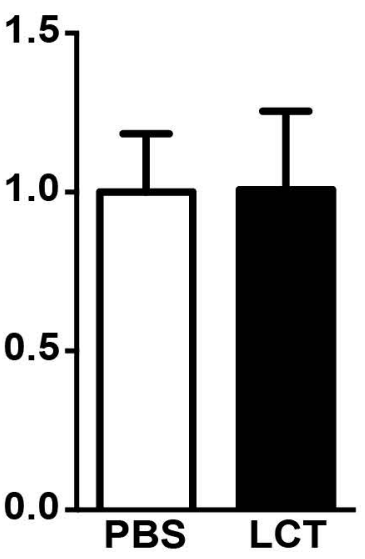



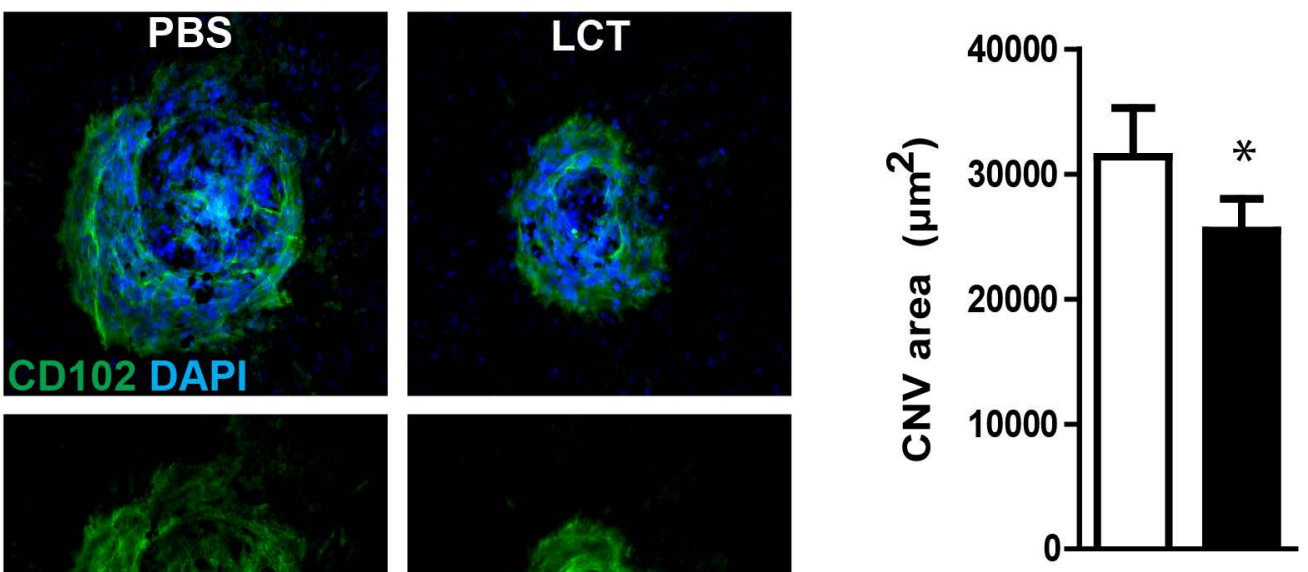

CD102

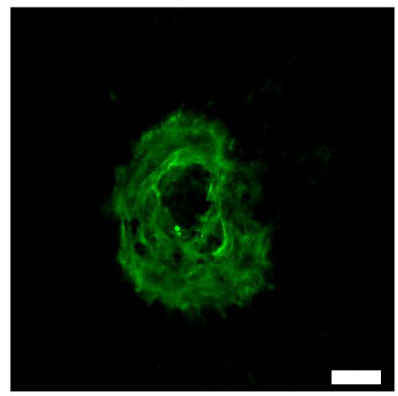

PBS

LCT $500 \mu \mathrm{M}$

Figure 6 

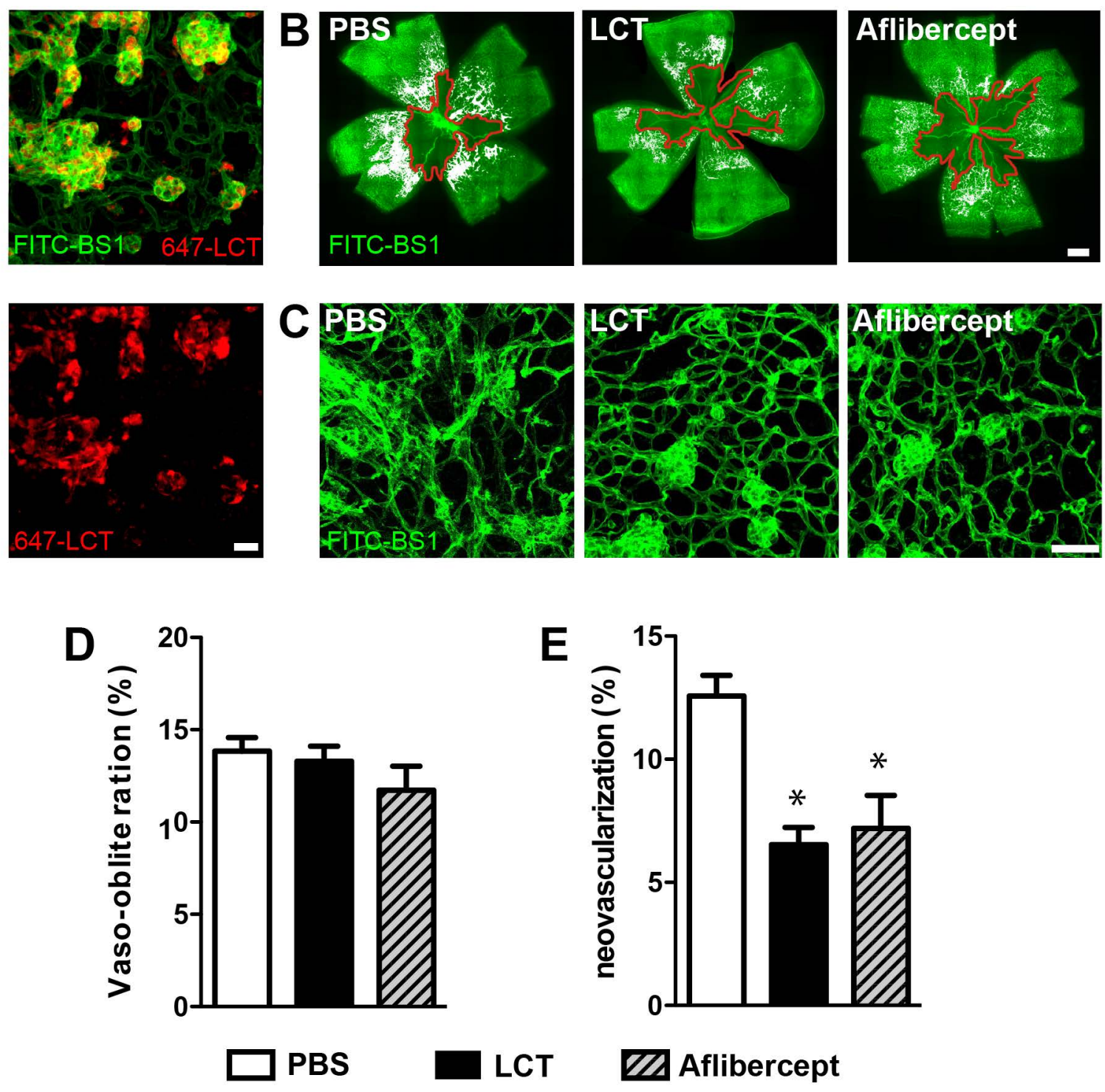

Figure 7 

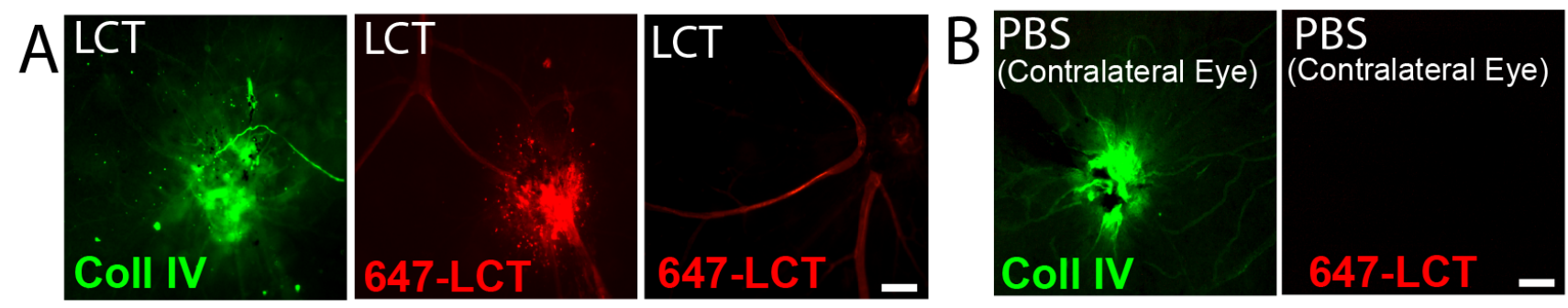

Supplementary Figure 1: LCT is not detected in the contralateral PBS -injected eye

Microphotographs of laser-induced CNV lesions at D7 on retinal flatmounts after intravitreal injections on D4 of LCT covalently conjugated to an Alexa Fluor-647 dye (647-LCT) in the right eye (A) and PBS in the contralateral left eye (B). All retinas were co-stained with collagen-IV antibody (green). Scale bars in B-E $=100 \mu \mathrm{m}$. 


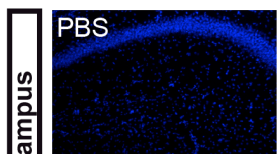

LCT

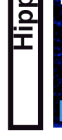

DAP
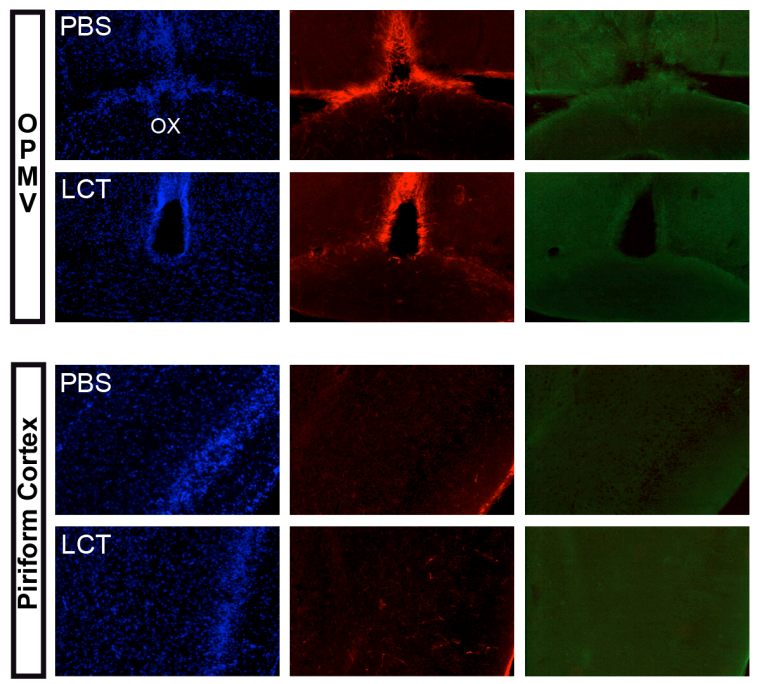
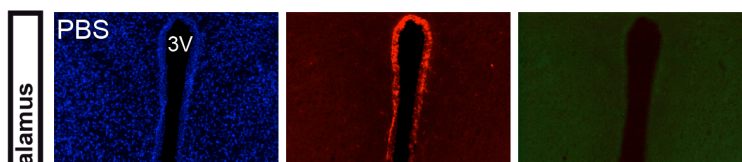

LCT

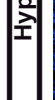

DAPI
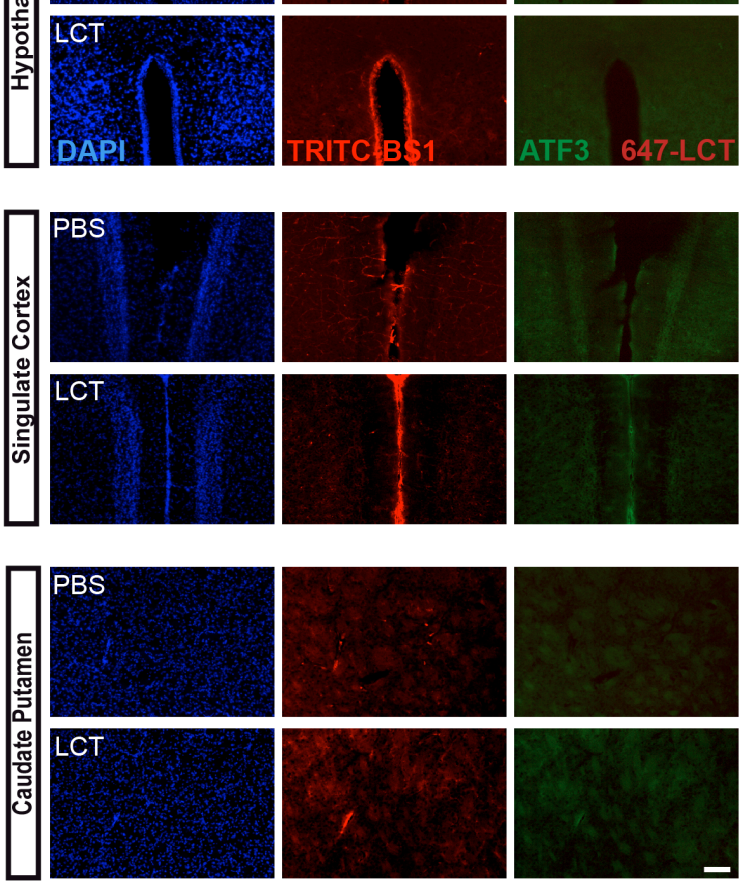

Supplementary figure 2: LCT is not detected in the mouse brain and does not trigger central neuronal injury after intravitreal injection.

Microphotographs of free floating sections of various brain structures 3 days after an intravitreal injection of LCT covalently conjugated to an Alexa Fluor-647 dye (647-LCT) in the right eye and PBS in the contralateral left eye (LCT) or PBS in both eye (PBS). Mice were subjected to laser induced CNV on D0. Free floating sections were stained with DAPI (blue), TRITC-BS1 lectin (red) and immunostained with ATF3 (green). VMPO: ventromedial preoptic nucleus, Ox: optic chiasm; 3V: third ventricle. Scale bar: $100 \mu \mathrm{m}$. 